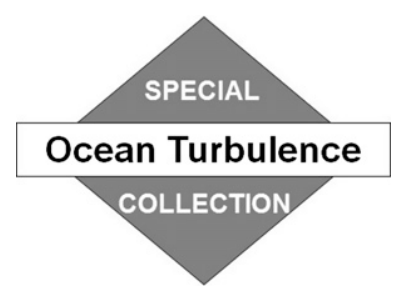

\title{
Time Scales of Southern Ocean Eddy Equilibration ${ }^{0}$
}

\author{
ANIRBAN SINHA \\ Applied Physics and Applied Mathematics Department, Columbia University, New York, New York \\ RYAN P. ABERNATHEY \\ Department of Earth and Environmental Sciences, Columbia University, New York, New York
}

(Manuscript received 14 February 2016, in final form 30 June 2016)

\begin{abstract}
Stratification in the Southern Ocean is determined primarily by a competition between westerly winddriven upwelling and baroclinic eddy transport. This study investigates the time scales of equilibration of the Southern Ocean in response to changing winds through an idealized channel model. An analytical framework describing the energetic pathways between wind input, available potential energy (APE), eddy kinetic energy (EKE), and dissipation provides a simple theory of the phase and amplitude response to oscillating wind stress. The transient ocean response to variable winds lies between the two limits of Ekman response (high frequency), characterized by the isopycnal slope responding directly to wind stress, and "eddy saturation" (low frequency), wherein a large fraction of the anomalous wind work goes into mesoscale eddies. The crossover time scale is the time scale of meridional eddy diffusive transport across the Antarctic Circumpolar Current (ACC) front. For wind variability with a period of 3 months (high-frequency forcing), the relative conversion of wind work to APE/EKE is 11, while for a period of 16 years (low-frequency forcing), the relative conversion to APE/EKE reduces to 3 . The system's frequency response is characterized by a complex transfer function. Both the phase and amplitude response of EKE and APE predicted by the linear analytic framework are verified using multiple ensemble experiments in an eddy-resolving (4-km horizontal resolution) isopycnal coordinate model. The results from the numerical experiments show agreement with the linear theory and can be used to explain certain features observed in previous modeling studies and observations.
\end{abstract}

\section{Introduction}

On account of the Southern Ocean's unique geometry, the Antarctic Circumpolar Current (ACC) is, compared to other regions, a dynamically unique ocean current, with large zonal transport and deep stratification (Rintoul and Naveira Garabato 2013). In the contemporary theory (e.g., Johnson and Bryden 1989; Marshall and Radko 2003; Abernathey and Cessi 2014),

Supplemental information related to this paper is available at the Journals Online website: http://dx.doi.org/10.1175/ JPO-D-16-0041.s1.

Corresponding author address: Anirban Sinha, Applied Physics and Applied Mathematics Department, Columbia University, 200 S. W. Mudd Building, MC 4701, 500 W. 120th Street, New York, NY 10027. E-mail: as4479@columbia.edu the steady-state equilibrium for ACC stratification and overturning circulation is determined by a competition between buoyancy transport by wind-driven upwelling and baroclinic eddy transport. Strong westerly winds over the Southern Ocean steepen the meridional isopycnal slopes through Ekman upwelling, which causes baroclinic instability, leading to vigorous eddies. These eddies transport heat poleward and momentum vertically, flattening the isopycnal slopes. The sensitivity of the isopycnal slopes and the associated thermal wind transport to the wind forcing has been studied extensively through many eddy-permitting and eddy-resolving models (Hallberg and Gnanadesikan 2001; Henning and Vallis 2005; Hallberg and Gnanadesikan 2006; Meredith and Hogg 2006; Hogg et al. 2008; Viebahn and Eden 2010; Farneti and Delworth 2010; Treguier et al. 2010; Abernathey et al. 2011; Meredith et al. 2012; Morrison and Hogg 2013; Munday et al. 2013; Abernathey and 


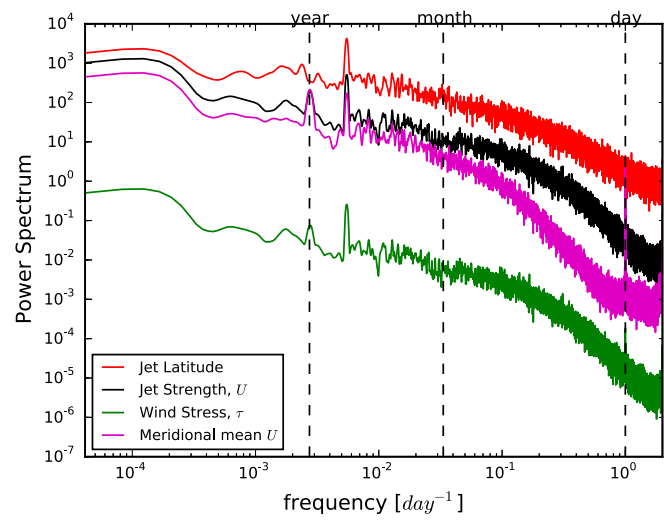

(a) NCEP-NCAR

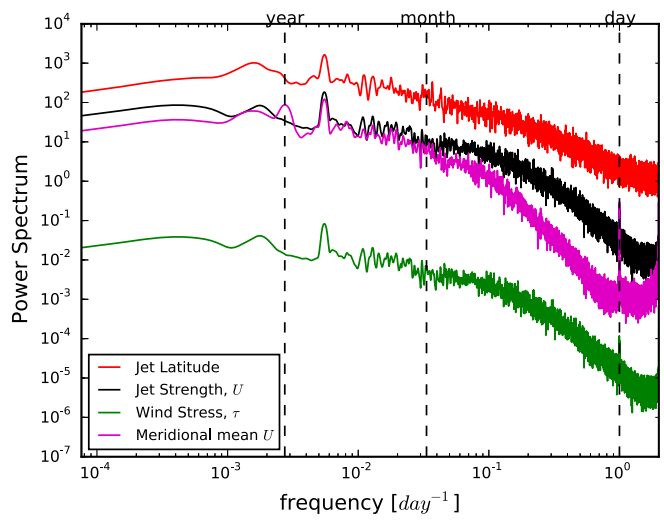

(b) ERA- Interim

FIG. 1. Power spectral density of the jet latitude, jet strength, wind stress, and mean zonal wind over the latitude region $35^{\circ}-70^{\circ} \mathrm{S}$ for the (a) NCEP-NCAR reanalysis (for the period 1948-2013) and (b) ERA-Interim (for the period 1979-2013) datasets with 6-hourly data. The power spectrum is computed using multitaper spectral methods and has been smoothed with a 10-point, smoothing Hanning window. Note the dominant peak is the semiannual time scale (1/2 yr). This is the well-known semiannual oscillation (Taschetto et al. 2007).

Cessi 2014; Pennel and Kamenkovich 2014; Hogg et al. 2015; Munday and Zhai 2015; Wilson et al. 2015; Marshall et al. 2016). These studies have collectively shown that mesoscale eddies strongly damp the sensitivity of isopycnal slopes to decadal-scale wind changes, a phenomenon often called "eddy saturation." Eddy saturation requires an approximately linear proportionality between winds and eddy kinetic energy (Meredith et al. 2012). Most of the work cited above has emphasized the long-term "equilibrium sensitivity" of the Southern Ocean to wind changes. Here, we investigate the physical processes and time scales involved in the transient response to time-variable winds.

The most studied transient variation in Southern Ocean winds is the well-known increase in the southern annual mode over the past $30 \mathrm{yr}$ (Marshall 2003). During this time, the maximum zonal wind stress forcing has increased by at least $20 \%$ (Swart and Fyfe 2012). Consistent with the eddy saturation concept, estimates from observations and models have suggested that there has been little change in ACC transport over this period (Cunningham et al. 2003; Hallberg and Gnanadesikan 2006; Boning et al. 2008; Chidichimo et al. 2014), although there is some evidence for small but nonzero dependence of ACC transport response to the SAM on interannual time scales, with small (near zero) lag (Meredith et al. 2004). However, the winds exhibit variability on all time scales. To characterize this variability, in Fig. 1 we present frequency power spectra of the surface zonal jet speed, jet latitude, and wind stress from the high-frequency (6 hourly) NCEP-NCAR (Kalnay et al. 1996; for the period 1948-2013) and ERA-Interim (Dee et al. 2011; 1979-2013) reanalyses. The jet was identified using the method described in Barnes and Polvani (2013) and Grise and Polvani (2014). We also show the meridionally averaged zonal wind over the latitude belt $35^{\circ}-70^{\circ} \mathrm{S}$. The spectra are red, with significantly more power at lower frequencies. We also observe a clear peak around the $1 / 2$-yr period, the wellknown semiannual oscillation (Taschetto et al. 2007). These spectra make it clear that the Southern Ocean experiences significant variability in wind forcing on time scales from monthly to decadal. Does the eddy saturation principle apply across all these scales?

From analyzing satellite altimetry data, Meredith and Hogg (2006) observed that the Southern Ocean eddy kinetic energy (EKE) exhibits anomalously high values around 2-3 yr after a transient peak in zonal wind stress (see also Hogg et al. 2015). Such a scenario was investigated with a quasigeostrophic (QG), eddy-resolving ocean model (Meredith and Hogg 2006; Hogg et al. 2008), which exhibited the same 2-3-yr lagged EKE response to a transient wind perturbation. To explain the lag, the authors proposed a mechanism wherein the initial wind energy perturbation is stored as potential energy (PE) and slowly transferred to EKE over several years. By considering a step function wind increase in a similar QG model, Meredith et al. (2012) showed that, after a similar 2-3 equilibration period, the EKE eventually reached a linear proportionality to the winds, as necessary for eddy saturation. A recent study (Patara et al. 2016) with a high-resolution $\left(1 / 2^{\circ}\right.$ in the ACC) ocean model revealed that EKE exhibits this lagged response to wind stress anomalies but with significant spatial nonuniformity. The response of EKE variability to wind stress is masked by stochastic variability in 
certain regions of the ACC. An obvious question is what determines this 2-3-yr time scale, which is much longer than the baroclinic growth rate from linear stability analysis (Tulloch et al. 2011)?

There have been several other recent studies of the transient response problem. Using a global eddy-permitting ocean circulation model driven with both idealized and realistic wind forcing, Langlais et al. (2015) examined the ACC response to interannual wind variability and observed that the response (barotropic transport, baroclinic transport, and EKE) depended on the spatial pattern of the changes in wind forcing. An enhancement of the westerlies over the ACC belt in their numerical model led to an increase of both barotropic and baroclinic transport, with lagged enhancement of EKE, while an increase in wind forcing near Antarctica was observed to drive a largely barotropic change in transport, with little change in eddy activity. Finally, Wilson et al. (2015) explored further the mechanisms of the transient response to a linear increase in wind stress using ensemble runs of a three-layer idealized Southern Ocean QG model. As with prior QG models, they reported no increase in circumpolar volume transport in response to the increased wind stress. By calculating the power spectrum of the energy budget, Wilson et al. (2015) showed that different terms dominated at low versus high frequencies, suggesting that qualitatively different regimes are possible at different time scales. Our spectral approach is similar to theirs.

In this paper, we seek to understand the nature of the Southern Ocean response to different frequencies of forcing. We argue that the response to changing winds lies between the two limits of Ekman (fast) response and an eddy-saturated (slow) response and that the mechanisms that govern the response are different in these two regimes. In the limit of high-frequency forcing, the ocean responds by converting all the excess wind work into available potential energy by Ekman upwelling and tilting isopycnals, and the isopycnals adjust before energy can be transferred effectively into the eddies. In the lowfrequency limit (slow limit), most of the additional wind work is converted into eddy kinetic energy via baroclinic eddies. At intermediate forcing frequencies, the response goes through a regime shift, determined by the effectiveness of the eddies at extracting energy through baroclinic instability. Using the energy budget, we develop a quantitative theory for the ACC response to time-varying winds as a function of forcing frequency that includes the wind power input, conversion to EKE via baroclinic instability, and dissipation. This theory makes specific predictions regarding the phase and amplitude response of isopycnal slopes and EKE, which we then test using idealized, adiabatic, eddy-resolving numerical simulations.
The paper is organized as follows: In section 2, we derive a simple conceptual model of the energy pathways in the ACC and introduce a "transfer function" approach to obtain solutions to the simple system of equations. In section 3 , we describe the suite of numerical experiments used, describe the analysis employed, and discuss the results. The results from the numerical simulations are compared quantitatively with the conceptual model in section 4 . We summarize the results and discuss the implications of the present work in section 5 .

\section{Conceptual model: Energy pathways}

In this section, we derive a theoretical framework for understanding the energetic pathways involved in the Southern Ocean response to variable wind forcing. In an adiabatic ocean, conservation of energy dictates that whatever energy is input into the system via wind work has to be used either to raise the available potential energy (APE) or to increase kinetic energy, which can be dissipated through boundary friction. Anomalous Ekman pumping acts directly to advectively tilt isopycnals. APE is proportional to the mean isopycnal slope and also, via thermal wind, to the baroclinic zonal transport $U_{T}$ (Abernathey and Cessi 2014). EKE is expected to respond more slowly, since eddies derive their energy from APE via baroclinic instability.

For our present study, we consider an adiabatic channel flow forced at the surface by oscillating winds and ignore all diapycnal fluxes of buoyancy and surface buoyancy fluxes. By doing so, we essentially eliminate thermodynamics, isolating the dynamical response of the ocean. Of course, in the real Southern Ocean, timedependent thermodynamic processes will also be involved in the response to changing winds (e.g., Ferreira et al. 2015), which could be investigated by applying the same approach in future studies. Here, the focus is on the behavior of mesoscale turbulence.

Under quasigeostrophic scaling, the energy budget (Lorenz 1960; Holland 1978) for the system can be expressed as

$$
\begin{aligned}
& \frac{d(\mathrm{APE})}{d t}=W-C, \\
& \frac{d(\mathrm{EKE})}{d t}=C-D,
\end{aligned}
$$

where $W=$ wind power input $=\left(1 / \rho_{0}\right) \iint(\tau \cdot \mathbf{u}) d A$,

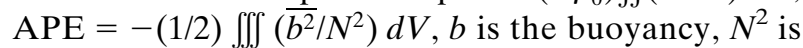
the background buoyancy frequency (representing the background stratification), $\left.\mathrm{EKE}=(1 / 2) \iiint \overline{\left(u^{2}+v^{\prime 2}\right.}\right) d V$, 
$\left.C=-\iiint \overline{\left(w^{\prime} b^{\prime}\right.}\right) d V$ is the conversion term, and $D$ is the rate of frictional dissipation. Here, primes denote the deviation from time-mean quantities. A derivation of these equations is given in the appendix. In this system, the rate of change of APE is directly proportional to the wind power input, and energy is removed from APE into EKE by means of the conversion term $C$, which acts as a sink of energy in the APE budget and a source in the EKE budget; $D$ is the rate at which energy is dissipated by frictional drag (predominantly bottom friction).

Standard mesoscale parameterizations (Gent and McWilliams 1990; Gent et al. 1995; Tandon and Garrett 1996) suggest that the conversion rate should be proportional to APE itself. The constraint of adiabatic eddy fluxes (Marshall and Radko 2003) requires that

$$
\overline{w^{\prime} b^{\prime}}=-\overline{v^{\prime} b^{\prime}} \frac{\overline{b_{y}}}{N^{2}} .
$$

Using a Gent-McWilliams (GM)-type (Gent and McWilliams 1990; Gent 2011) closure $\left(-\overline{\boldsymbol{v}^{\prime} b^{\prime}}=K_{\mathrm{GM}} \overline{b_{y}}\right)$, we can rewrite the conversion term as

$$
\overline{w^{\prime} b^{\prime}}=K_{\mathrm{GM}} \frac{\bar{b}_{y}^{2}}{N^{2}} .
$$

From a first-order Taylor series expansion, we can also write $b \simeq \bar{b} \simeq(\partial \bar{b} / \partial y) y$. This implies

$$
\mathrm{APE}=-\frac{1}{2} \iiint_{d V} \frac{\overline{[y(\partial \bar{b} / \partial y)]^{2}}}{N^{2}} .
$$

Now since APE $\propto{\overline{b_{y}}}^{2} / \overline{b_{z}}$, we can rewrite the conversion term as

$$
-\iiint_{d V} \overline{w^{\prime} b^{\prime}} \approx \frac{K_{\mathrm{GM}}(\mathrm{APE})}{L_{y}^{2}} .
$$

This closure employs a constant eddy diffusivity $K_{\mathrm{GM}}$ that is independent of EKE, neglecting potential eddy feedbacks (which we will consider later). As a result, the conversion of APE to EKE is linearly proportional to the APE. In fact, a large motivation for the GentMcWilliams scheme is to extract APE from the flow in a manner consistent with baroclinic instability (Tandon and Garrett 1996; Chelton et al. 1998; Stammer 1998; Smith 2007). We will henceforth refer to this mechanism of conversion of APE to EKE at a rate proportional to the APE reservoir as linear baroclinic conversion. The time scale for this mechanism is given by the constant of proportionality $c=K_{\mathrm{GM}} / L_{y}^{2}$. Plugging in representative values for the ACC, $K_{\mathrm{GM}}=1000 \mathrm{~m}^{2} \mathrm{~s}^{-1}$ and $L_{y}=10^{6} \mathrm{~m}$, we obtain $c=10^{-9} \mathrm{~s}^{-1}$ or roughly $(3 \mathrm{yr})^{-1}$. Note this is very close to the equilibration time scale found empirically by previous authors (Hogg et al. 2008; Meredith et al. 2012).

For ease of representation, we denote $f(t)$ as the external forcing (wind power input), $P(t)$ as the APE, and $K(t)$ as the EKE. For now, we also assume that EKE is dissipated linearly (i.e., linear bottom drag), with a frictional inverse time scale $r$. For this case, we can express the energy budget (2) as

$$
\begin{aligned}
& \frac{d P(t)}{d t}=f(t)-c P(t), \quad \text { and } \\
& \frac{d K(t)}{d t}=c P(t)-r K(t) .
\end{aligned}
$$

Motivated by an analogy to linear, time-invariant electrical signals (Girod et al. 2001), we derive the transfer functions for the outputs $P(t)$ and $K(t)$, given a time periodic input signal $f(t)$ with input frequency $\omega$. We take the Fourier transform of (6) and (7), thereby assuming periodic solutions: $f=\hat{f} e^{i \omega t}, P=\hat{P} e^{i \omega t}$, and $K=\hat{K} e^{i \omega t}$, where the hatted variables are the complex Fourier amplitudes. (The initial-value problem can instead be solved with a Laplace transform.) The energy equations become

$$
\begin{aligned}
i \omega \hat{P} & =\hat{f}-c \hat{P}, \quad \text { and } \\
i \omega \hat{K} & =c \hat{P}-r \hat{K} .
\end{aligned}
$$

The output solutions in the frequency domain are given by

$$
\begin{aligned}
& \hat{P}(\omega)=|\hat{P}(\omega)| e^{i \phi_{P}(\omega)}=\frac{c-i \omega}{c^{2}+\omega^{2}} \hat{f}(\omega), \text { and } \\
& \hat{K}(\omega)=|\hat{K}(\omega)| e^{i \phi_{K}(\omega)}=\frac{r c-\omega^{2}-i \omega(r+c)}{\left(r c-\omega^{2}\right)^{2}+\omega^{2}(r+c)^{2}} c \hat{f}(\omega) .
\end{aligned}
$$

The output functions $\hat{P}$ and $\hat{K}$ are complex; the modulus represents the amplitude of the response, and the phase describes the time lag between input and output. For a given input $\hat{f}$, the response is characterized by the gains $(|\hat{P} / \hat{f}|$ and $|\hat{K} / \hat{f}|)$ and phase shifts $\left(\phi_{P}\right.$ and $\left.\phi_{K}\right)$.

The gains and phase shifts for APE and EKE are calculated and plotted in Fig. 2 for different values of drag $r$. Both $\omega$ and $r$ are nondimensionalized by $c$. In the low-frequency limit $(\omega \ll c)$, the APE and EKE gain plateau to a constant maximum value. The maximum EKE gain is determined by the linear drag coefficient (Abernathey and Cessi 2014). As seen in Fig. 2, EKE saturates at the largest value for the smallest drag coefficient and equals the maximum APE when $r=c$, that is, when the frictional dissipation rate is the same as the 

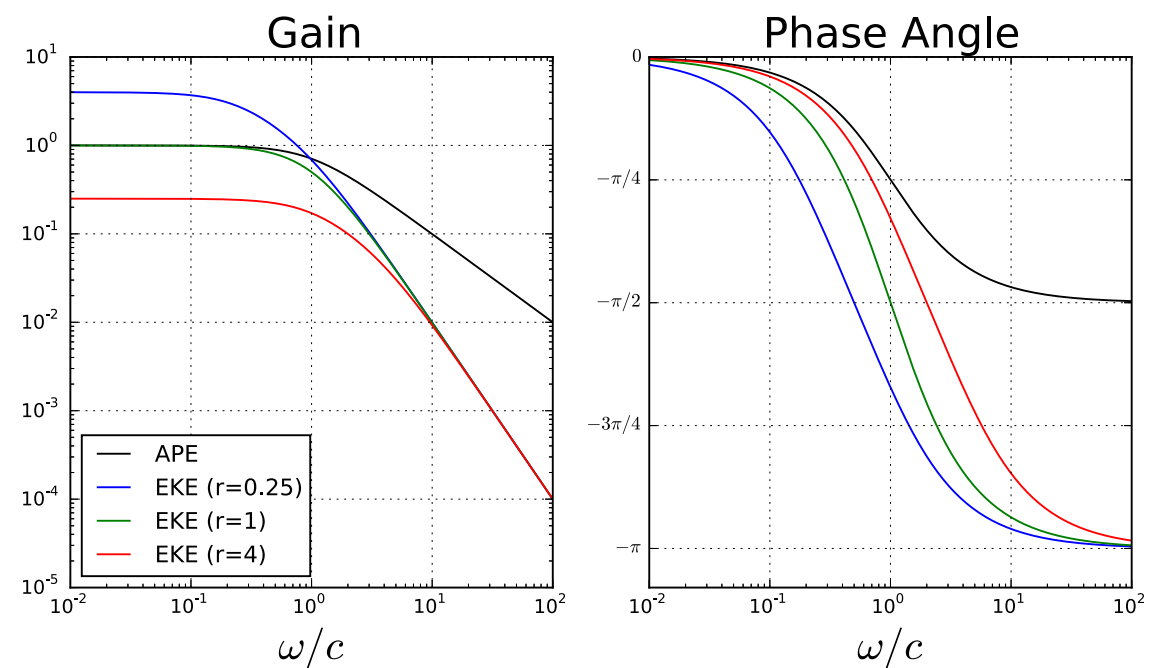

FIG. 2. The (left) gain and (right) phase shift angle of EKE and APE for the analytical model of time-dependent eddy compensation with no eddy feedback [(6) to (11)] as a function of nondimensionalized $\omega$. The parameter $r$ is also nondimensionalized by $c$. Note, in particular, the qualitative difference in behavior between the high frequencies $(\omega / c>1)$ and the low frequencies $(\omega / c<1)$.

rate of baroclinic conversion. In the high-frequency limit $(\omega \gg c)$, APE gain is proportional to $\omega^{-1}$, while EKE gain is proportional to $\omega^{-2}$. This means that highfrequency variability will have a much greater impact on APE than EKE. The high-frequency limit corresponds to Ekman pumping of the isopycnals up and down.

Since the conversion term in (7) is proportional to APE only (linear baroclinic conversion), this solution fails to capture an important nonlinear interaction: the feedback of the EKE on the conversion rate through enhanced eddy mixing. We can incorporate this feedback into the conversion term by invoking mixing length arguments (Ferrari and Nikurashin 2010; Klocker et al. 2012; Abernathey et al. 2013; Klocker and Abernathey 2014; Abernathey and Ferreira 2015). We replace the constant $K_{\mathrm{GM}}$ with an effective eddy coefficient (Abernathey and Ferreira 2015):

$$
K_{\text {eff }}=\Gamma_{\text {mix }} v_{\text {rms }} L_{\text {mix }},
$$

where $\Gamma$ is a constant mixing efficiency, and $L_{\text {mix }}$ is the mixing length, which is related to eddy length scales; $v_{\text {rms }}$ is related to EKE as $v_{\mathrm{rms}}=\left|v^{12}\right|^{1 / 2}=(\mathrm{EKE})^{1 / 2}$. Using this relationship, and assuming the mixing length remains constant, we modify the conversion term as

$$
C=\frac{K_{\mathrm{eff}}(\mathrm{APE})}{L_{y}^{2}}=k(\mathrm{APE})(\mathrm{EKE})^{1 / 2},
$$

where all the constants $\Gamma_{\text {mix }}, L_{\text {mix }}$, and $L_{y}$ are incorporated into the constant $k$ as $k=\Gamma_{\text {mix }} L_{\text {mix }} / L_{y}^{2}$. To enable tuning of this eddy feedback, we can allow a more general relationship between conversion and EKE by setting $C=k(\mathrm{APE})(\mathrm{EKE})^{\alpha}$. Here, $\alpha(0 \leq \alpha \leq 1)$ indicates the efficiency of the EKE feedback; a higher $\alpha$ indicates higher sensitivity of the conversion rate to EKE. Equivalently, we can accommodate different types of drag using $D=r^{*} K^{\beta}$, where $\beta$ depends on the drag power law used: for linear drag, $\beta=1$, and for quadratic drag, $\beta=3 / 2$.

To incorporate these potential nonlinearities, we modify (6) and (7) as

$$
\begin{aligned}
& \frac{d P}{d t}=f-k P K^{\alpha}, \quad \text { and } \\
& \frac{d K}{d t}=k P K^{\alpha}-r^{*} K^{\beta} .
\end{aligned}
$$

The steady-state solution (indicated by an overbar) is obtained by taking the time mean of (14) and (15):

$$
\begin{aligned}
\bar{f} & =k \overline{P K^{\alpha}}, \quad \text { and } \\
r^{*} \overline{K^{\beta}} & =k \overline{P K^{\alpha}} .
\end{aligned}
$$

If the fluctuations from the time-mean quantities are small, we can linearize by neglecting the time-varying correlations between EKE and APE and solve for the constants $k$ and $r^{*}$. A posteriori, based on the numerical simulations, this approximation is well justified for the high-frequency limit but less so at the low frequencies (see Fig. 9): 


$$
\begin{aligned}
k & =\frac{\bar{f}}{\bar{K}^{\alpha} \bar{P}}, \text { and } \\
r^{*} & =\frac{k \bar{P} \bar{K}^{\alpha}}{\bar{K}^{\beta}} .
\end{aligned}
$$

To apply the transfer function approach, a linear system of equations is required. Linearizing about the timemean state, we obtain

$$
\begin{aligned}
k P K^{\alpha}= & k\left(\bar{P}+P^{\prime}\right)\left(\bar{K}+K^{\prime}\right)^{\alpha} \\
\approx & k\left(\bar{P}+P^{\prime}\right) \bar{K}^{\alpha}\left(1+\alpha \frac{K^{\prime}}{\bar{K}}\right), \quad \text { and } \\
= & k \bar{P} \bar{K}^{\alpha}+k \alpha \bar{K}^{\alpha-1} \bar{P} K^{\prime}+k P^{\prime} \bar{K}^{\alpha} \\
& +k \alpha \bar{K}^{\alpha-1} P^{\prime} K^{\prime},
\end{aligned}
$$

where the time-varying part is denoted by primes. Neglecting higher-order terms, the time-varying equation can now be written as

$$
\begin{aligned}
& \frac{d P^{\prime}}{d t}=f^{\prime}-k P^{\prime} \bar{K}^{\alpha}-k \alpha \bar{P} \bar{K}^{\alpha-1} K^{\prime}, \quad \text { and } \\
& \frac{d K^{\prime}}{d t}=k P^{\prime} \bar{K}^{\alpha}+k \alpha \bar{P} \bar{K}^{\alpha-1} K^{\prime}-r^{*} \beta \bar{K}^{\beta-1} K^{\prime} .
\end{aligned}
$$

We define constants $c_{1}=k \bar{K}^{\alpha} \approx \bar{f} / \bar{P}$ and $c_{2}=k \alpha \bar{P} \bar{K}^{\alpha-1} \approx$ $\alpha \bar{f} / \bar{K}$ and $r=r^{*} \beta \bar{K}^{\beta-1} \approx \beta \bar{f} / \bar{K}$, giving

$$
\begin{aligned}
& \frac{d P^{\prime}}{d t}=f^{\prime}-c_{1} P^{\prime}-c_{2} K^{\prime}, \quad \text { and } \\
& \frac{d K^{\prime}}{d t}=c_{1} P^{\prime}+c_{2} K^{\prime}-r K^{\prime} .
\end{aligned}
$$

Note that this system of equations is very similar to (6) and (7), with a single extra term $\left(c_{2} K^{\prime}\right)$; upon setting $c_{2}=$ 0 , we get back our previous solution without eddy feedbacks. The sum $c_{1} P^{\prime}+c_{2} K^{\prime}$ now denotes the total rate at which energy is converted from APE to EKE. The first part of this conversion is $c_{1} P^{\prime}$, identical to the linear baroclinic conversion we obtained using a constant Gent-McWilliams coefficient in (5). This term continues to represent the influence of the isopycnal slopes themselves on the conversion rate. The second part $\left(c_{2} K^{\prime}\right)$ represents the eddy feedback due to the dependence of eddy mixing on EKE. The constant $c_{2}$ is dependent both on the steady-state APE as well as the steady-state EKE; $r$, the linearized frictional drag parameter, depends on the steady-state EKE in addition to the frictional drag coefficient $r^{*}$. The Fourier solution is obtained as

$$
\begin{aligned}
& \hat{K}=\frac{c_{1}}{-\omega^{2}+r c_{1}+i \omega\left(c_{1}-c_{2}+r\right)} \hat{f}, \quad \text { and } \\
& \hat{P}=\frac{\left(i \omega-c_{2}+r\right)}{-\omega^{2}+r c_{1}+i \omega\left(c_{1}-c_{2}+r\right)} \hat{f} .
\end{aligned}
$$

The gain and phase shift of the APE and EKE for different values of the constants are plotted in Fig. 3. The forcing frequency $\omega$ and the parameters $r$ and $c_{2}$ are normalized by the transfer rate $c_{1}$. Overall, the picture is similar to that given by the simpler model with no eddy feedback (Fig. 2). However, important differences also emerge, including that the eddy feedback has opened the door to behavior that resembles eddy saturation. The APE gain is no longer monotonically dependent on frequency; for certain parameter combinations (e.g., when $r=c_{2}$ ), it decreases as $\omega$ approaches zero. For weak friction, a near-resonance occurs, causing a peak in the gain for both APE and EKE.

Given the wide range of possible parameter combinations, and the parameter dependence on the mean state, it is not immediately obvious that combinations are relevant to the ACC in its present state. To test this analytic framework quantitatively, we now turn to numerical simulations that explicitly resolve the energy pathways described above. This allows us to estimate the parameters and verify the applicability of the transfer function approach.

\section{Numerical model}

With our numerical simulations, we attempt to realize the scenario described above as directly as possible. We conduct simulations using the isopycnal GOLD model of Hallberg and Gnanadesikan (2006; Hallberg 1997). The use of an isopycnal model enables the flow to be exactly adiabatic. However, in contrast to the QG models used in previous studies (e.g., Hogg et al. 2003, 2008; Meredith et al. 2012; Wilson et al. 2015), the model imposes no constraints on isopycnal slope.

We employ an idealized zonally periodic channel geometry $(3200 \mathrm{~km} \times 1600 \mathrm{~km} \times 4 \mathrm{~km})$ in Cartesian coordinates. A topographic ridge of $1 \mathrm{~km}$ height is present, blocking the abyssal zonal flow. The Rossby radius of deformation is of the order of $55 \mathrm{~km}$ in the middle of the domain. This configuration is identical to that described in Howard et al. (2015), with three stably stratified layers with densities 1025.0, 1030.0, and $1032.5 \mathrm{~kg} \mathrm{~m}^{-3}$. In contrast to global, eddy-resolving models, this idealized model can be run at high horizontal resolution $(4 \mathrm{~km})$ at a relatively low computational cost. The model solves the primitive equations of motion on a $\beta$ plane, in isopycnal coordinates, as described in Killworth and Nanneh (1994) and Ward and Hogg (2011), using three reducedgravity layers. We explore a range of forcing frequencies using a zonally invariant oscillating wind stress of the form

$$
\tau^{x}=\left[\tau_{0}^{x}+\tau_{1}^{x} \sin \left(\frac{2 \pi t}{T}\right)\right] \sin \left(\frac{\pi y}{L_{y}}\right)
$$



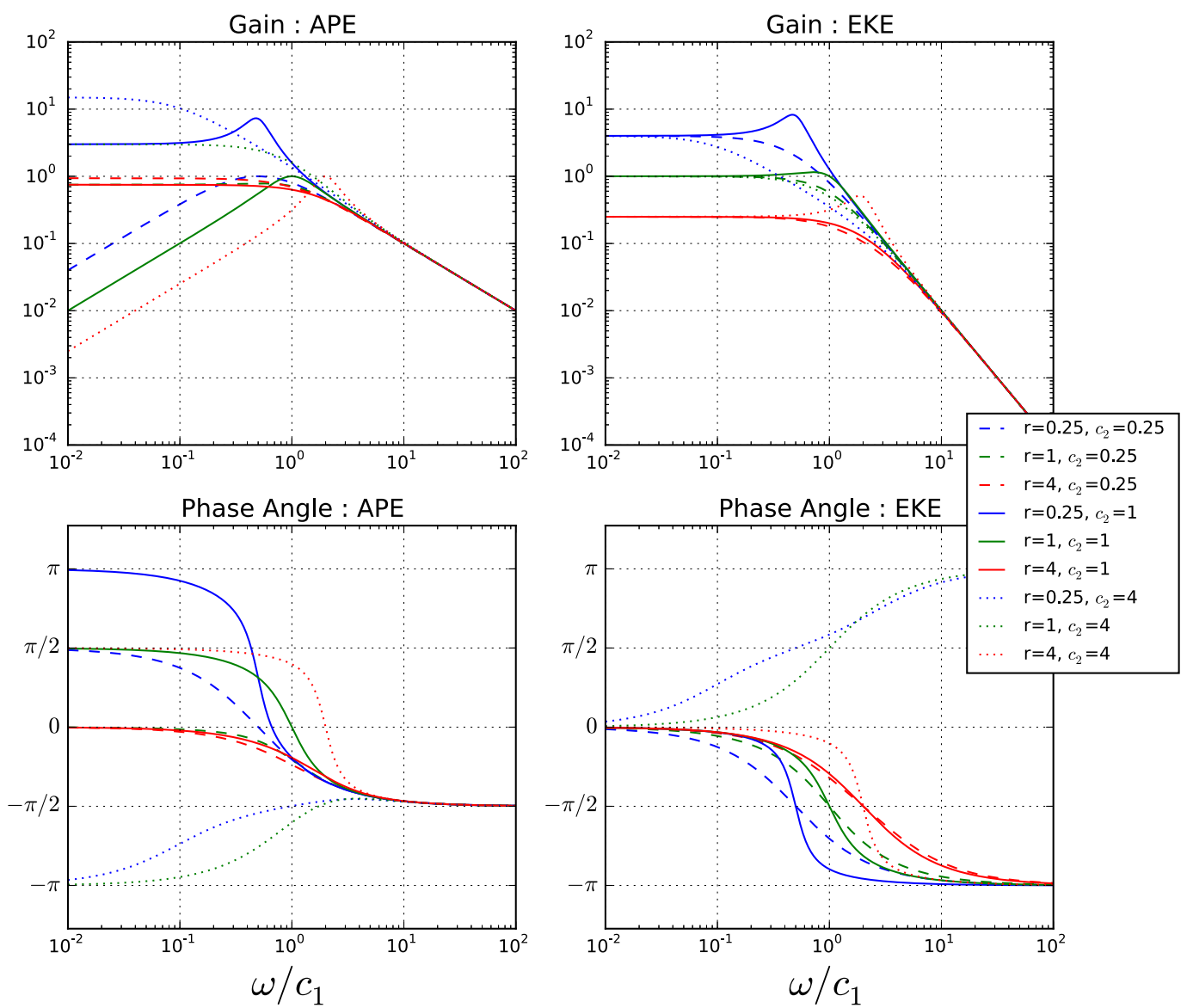

FIG. 3. The (top) gain and (bottom) phase shift angle of (left) APE and (right) EKE for the analytical model of time-dependent eddy compensation with the eddy feedbacks [(14) to (27)] as a function of nondimensionalized $\omega$ (normalized by $c_{1}$ ) for different values of eddy feedback rates $c_{2}$ and drag coefficients $r$. The parameters $r$ and $c_{2}$ are nondimensionalized by $c_{1}$.

where $T$ is the time period of the forcing frequency, and $L_{y}$ is the meridional extent of the channel. (Alternately we can represent the frequency of forced oscillations as $\omega=2 \pi / T$.) Figure 4 shows the magnitude of the wind stress and the configuration of the bottom topography. A snapshot of the model showing the three isopycnal layers and the relative vorticity in the top layer is also shown.

We conducted a series of ensemble numerical experiments with different forcing frequencies, with the forcing periods chosen as powers of $2 \mathrm{yr}$. For each forcing period $T, M$ ensemble members with different initial conditions are run for $N_{T}$ years, so as to have the same number of forcing cycles of integration for each set $\left[\left(N_{T} \times M\right) / T\right]$. The oscillating wind experiments consist of six sets with forcing periods $T \in\{1 / 4,1 / 2,1,2,4,8,16\} \mathrm{yr}$, along with an ensemble of control runs with time-invariant wind forcing. The details of the integration time and the number of ensembles used for each of these sets are provided in Table 1. In each of the variable wind forcing experiments, the peak wind stress of the half sine jet described in (28) is chosen to be $0.2 \mathrm{~N} \mathrm{~m}^{-2}$, and the magnitude of the oscillating part is chosen to be $0.1 \mathrm{~N} \mathrm{~m}^{-2}$. The control run equilibrates statistically after an 11-yr spinup. Initial conditions for each ensemble member were taken from this control.

From the model variables $(u, v, \eta, h$, and $\tau)$, the time series for EKE, APE, and wind work is constructed for each ensemble member. The time series information is analyzed in two different ways, as described in the next two subsections.

\section{a. Spectral analysis}

The frequency power spectrum provides information about the variability of the system at different time scales and is a natural starting point for analyzing the simulations. The power spectral density is computed for EKE, APE, and wind work from each ensemble member 


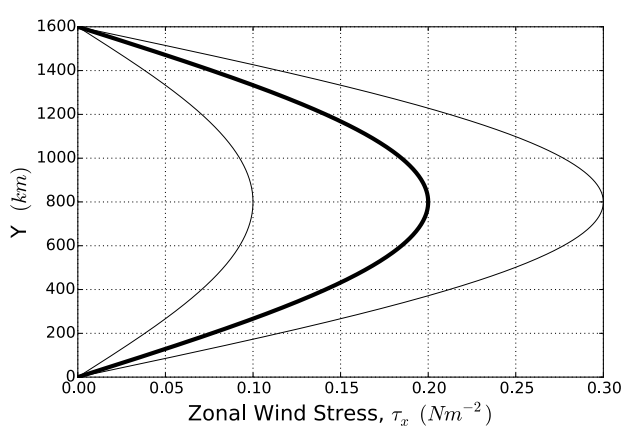

(a) wind stress

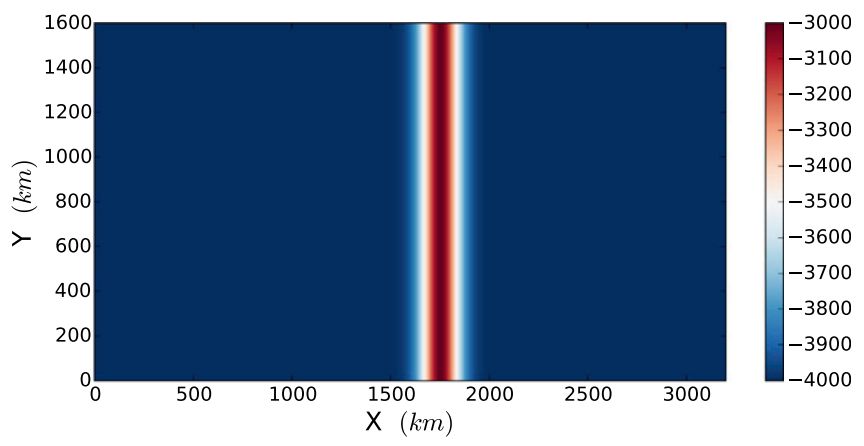

(b) bottom topography

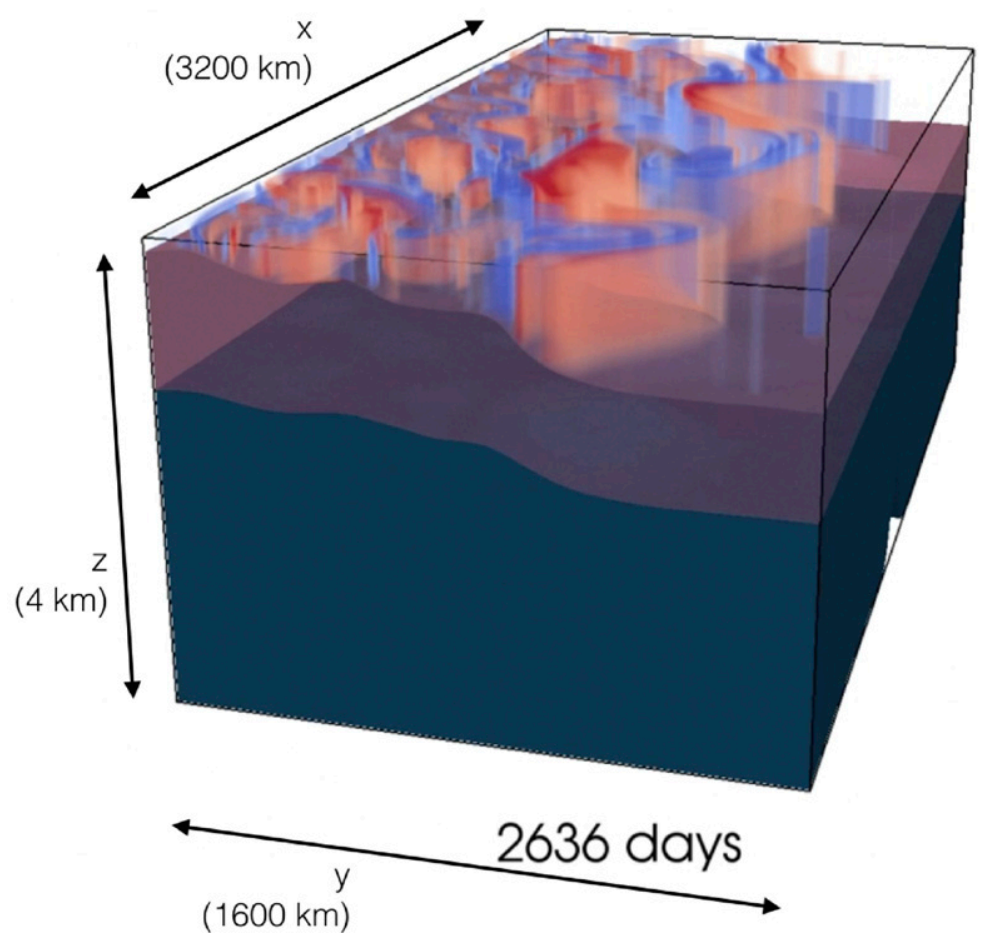

(c) snapshot of model spinup

FIG. 4. (a) Wind stress used for the numerical simulations with a steady part represented by the thick, black line and oscillating between the two thin lines with a frequency given by the forcing frequency of the particular experiment. (b) The bottom topography used in the numerical simulations showing a meridionally symmetric Gaussian ridge running down the middle of the domain. (c) Snapshot of the model spinup showing the isopycnal layers and the relative vorticity computed from the $u$ and $v$ fields for the top layer. An animation of the model during spinup can be viewed in the supplemental material.

using the multitaper spectral method (Thomson 1982, 2007; Millman and Brett 2007) and then averaged across ensembles. ${ }^{1}$

\footnotetext{
${ }^{1}$ While multitapering reduces estimation bias compared to ensemble averaging by obtaining multiple independent estimates from the same sample, the use of ensembles is computationally more efficient than one long simulation since the ensembles can be run in parallel.
}

The power spectral density for each variable is shown in Fig. 5 along with the ensemble standard deviation. Each set of experiments $(T \in\{1 / 4,1 / 2,1,2,4,8,16\}$ yr and steady wind forcing) is shown in a different color. Unsurprisingly, we see a clear peak in each spectrum at the corresponding forcing frequency. The wind oscillation amplitude is exactly the same for each forcing frequency, and consequently the maximum power spectral density in the wind work input is also roughly the same 
TABLE 1. Table showing the different numerical experiments performed.

\begin{tabular}{ccccc}
\hline \hline $\begin{array}{c}\text { Forcing } \\
\text { period }(\mathrm{yr})(T)\end{array}$ & $\begin{array}{c}\text { Number of } \\
\text { ensembles }(M)\end{array}$ & $\begin{array}{c}\text { Number of years } \\
\text { of integration } \\
\text { per ensemble }\left(N_{T}\right)\end{array}$ & $\begin{array}{c}\text { Sampling } \\
\text { frequency (days) }\end{array}$ & $\begin{array}{c}\text { Number of forcing } \\
\text { cycles }\left[\left(N_{T} \times M\right) / T\right]\end{array}$ \\
\hline $1 / 4$ & 4 & 15 & 2 & 240 \\
$1 / 2$ & 4 & 25 & 5 & 200 \\
1 & 4 & 50 & 5 & 200 \\
2 & 8 & 50 & 5 & 200 \\
4 & 8 & 100 & 10 & 200 \\
8 & 8 & 200 & 20 & 200 \\
16 & 4 & 400 & 40 & - \\
Steady & 4 & 50 & 5 & - \\
\hline
\end{tabular}

across the experiments. However, there is a slight decrease in peak wind work with increasing forcing frequency. The wind work is given by the product of the wind stress and the surface zonal-mean velocity. The slightly higher wind power input for low forcing frequencies can be attributed to a correlation between the forcing frequency and internal variability of the zonalmean flow. In fact, even the steady wind power spectrum shows higher wind power input at low frequencies.

APE and EKE clearly have different responses at different forcing frequencies. A striking feature is the lack of any observable peaks in EKE for high-frequency forcing $(T<1 \mathrm{yr})$. At low frequencies, in contrast, strong EKE peaks emerge. This behavior is in agreement with the analytical model, which predicted decreased EKE gain at high frequencies. (As a side note, secondary peaks are observed in the APE power spectral density at higher multiples of the forcing frequency.)

The magnitudes of the peaks in EKE, APE, and wind work are all different for the different cases. To assess the response of EKE and APE, we examine their peak amplitudes normalized by the peak amplitude of wind
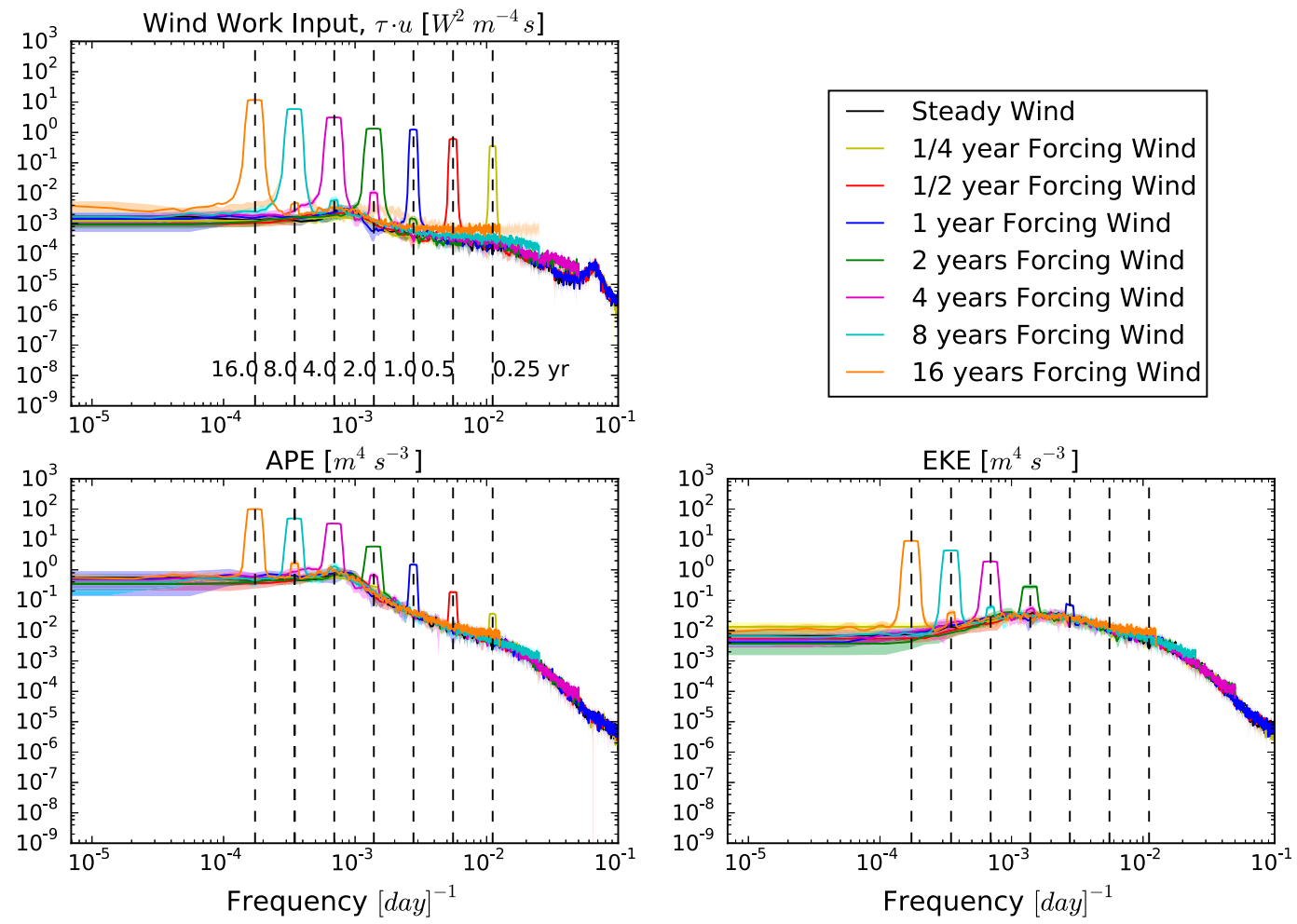

FIG. 5. Power spectral density of wind work input, APE, and EKE for the different forcing experiments. Note that the spectra are dominated by peaks at the corresponding forcing frequencies. Also note the lack of any observable peaks in EKE at the highest frequencies $(T<1 \mathrm{yr})$. 


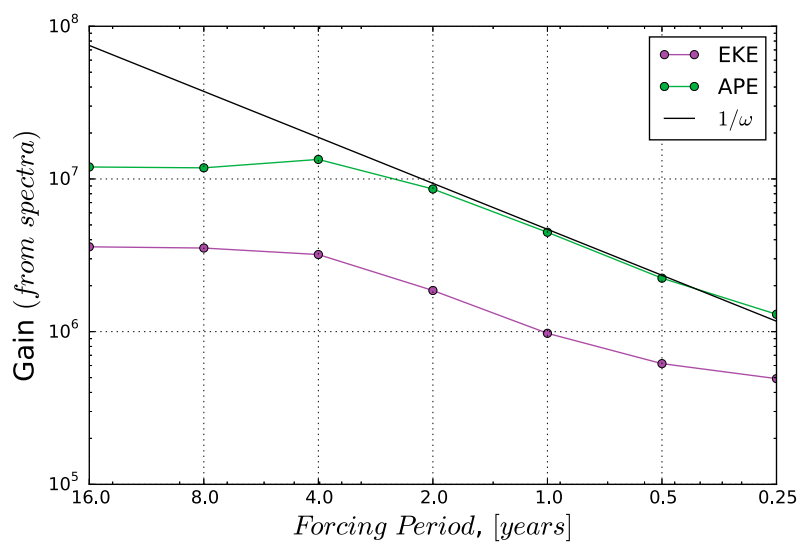

FIG. 6. Amplitude of oscillation for EKE and APE, computed from the power spectral densities of EKE and APE, normalized with the maximum amplitude of the wind work for each of the variable wind forcing experiments as a function of the forcing frequency.

work, that is, the gain, for each forcing frequency in Fig. 6. The $1 / \omega$ line is included to show that APE is inversely proportional to $\omega$ in the limit of high forcing frequency. In this respect as well, the diagnosed gain is in agreement with the analytical model. A more quantitative evaluation of the theory is presented in the next section.

Although our theory focused on the energy cycle, the zonal transport is also a quantity of interest. We diagnose time series of the total, barotropic, and baroclinic components of the zonal transport and calculate the power spectral density of these three quantities (shown in Fig. 7). The comparison of the spectra reveals that the lowfrequency transport variability is dominated by the baroclinic component, while the high-frequency variability is barotropic. The peaks in the baroclinic transport at the forcing frequencies are much stronger than those of the barotropic transport. The background slope of the power spectral density of baroclinic transport resembles the background slope of EKE, while the barotropic transport has an oppositely sloped spectrum for $T>1 / 4 \mathrm{yr}$ and displays a broad peak near the monthly time scale.

The spectral analysis reveals useful information about the qualitative nature of the amplitude response in the EKE and APE to variable wind forcing. The power spectra show that the response to oscillating winds is generally characterized by a peak at the corresponding forcing frequency, with little or no peak in EKE for high forcing frequency and relatively smaller APE response to low forcing frequency. To obtain a more mechanistic description of this response, we now perform a "composite analysis" to examine both the phase and amplitude response of the forced variability.

\section{b. Composite analysis}

For a periodic wind forcing at period $T$, the composite in wind work, EKE and APE is constructed by treating each $T$ periodic segment in the time series as an independent signal of length $T$ and then taking the mean over all such $T$ periods present in the time series and over all the $M$ composite members. Thus, for $M$ ensemble members and $N_{T}$ years of integration, this gives us $\left(M \times N_{T}\right) / T$ independent signals of length $T$ over which we take the mean and standard deviation to give the composite signal and the error in its estimation. This procedure effectively reduces the original noisy signal into a smooth periodic signal with period $T$. This is comparable to isolating a single frequency from the power spectrum. [Such an approach is common, e.g., in characterizing the annual cycle (Qiu et al. 2014).] A schematic of the composite approach is shown in Fig. 8.

Figure 9 shows the composite mean and standard deviation of EKE, APE, and wind work for each forcing frequency. An obvious feature is that the amplitude of

Zonal Transport $\left[m^{6} s^{-1}\right]$
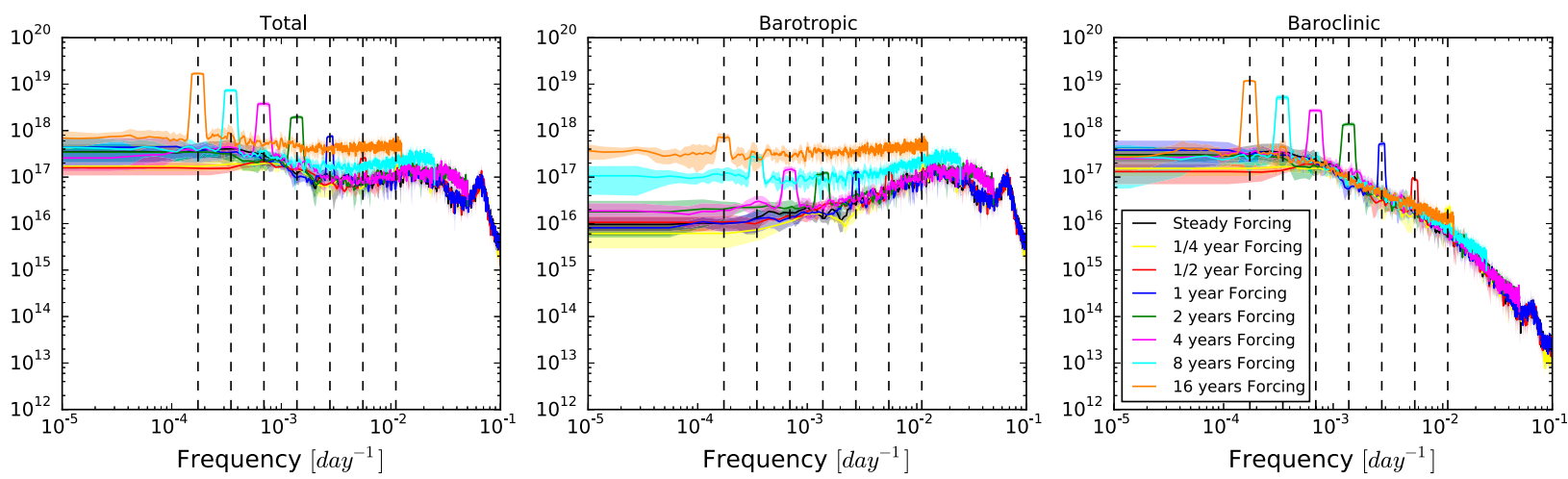

FIG. 7. Power spectral density for the total zonal transport and the barotropic and baroclinic parts for the different experiments. 


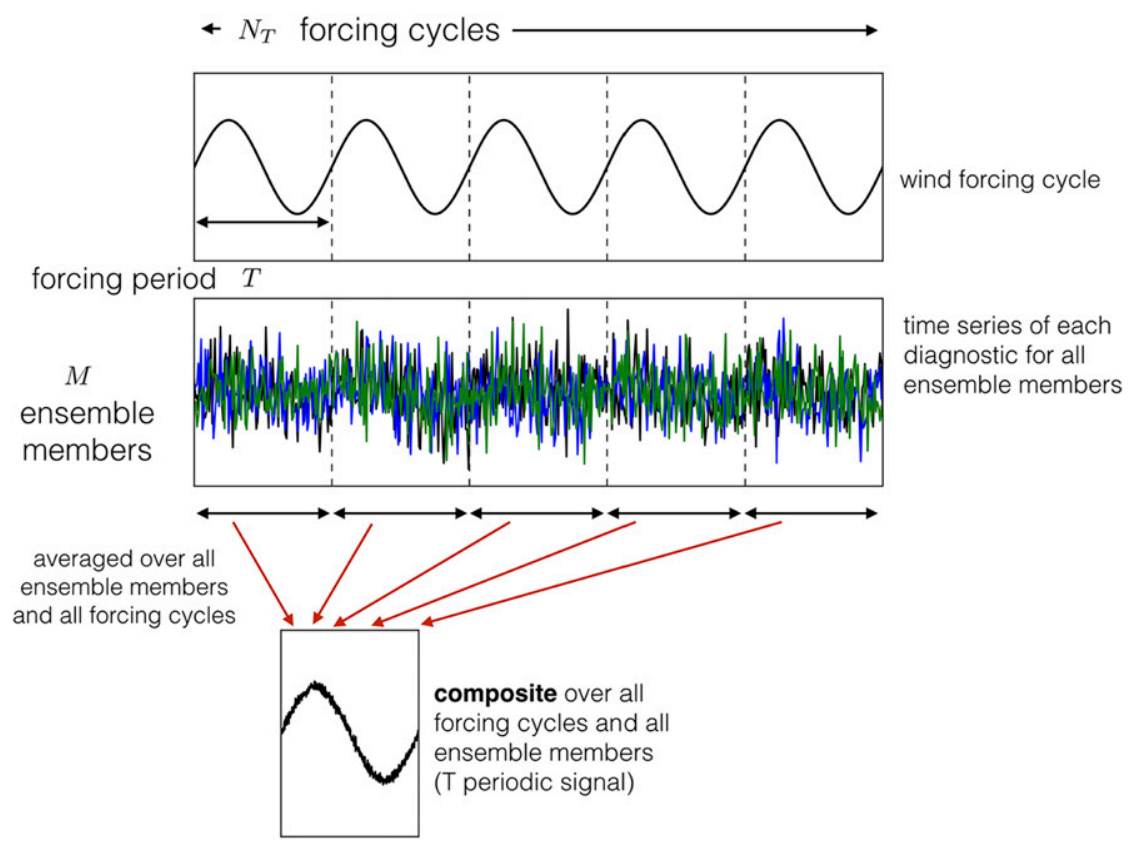

FIG. 8. Schematic describing the method used for calculating the composite of a particular variable for the composite analysis.

oscillation in EKE is very small (almost absent in the $1 / 4-y r$ forcing experiment) and increases as the forcing frequency decreases, in agreement with the results from the spectral analysis. As the forcing period increases, the amplitude of the response increases as well and falls more into phase with the forcing.

The amplitude of oscillation of EKE and APE is computed by subtracting the mean from the composite signal and then calculating the root-mean-square (rms). The gain for APE and EKE is given by normalizing by the amplitude of the wind work composite. This may differ slightly from the gain found through spectral analysis because the composite analysis explicitly removes the internal variability at the forcing frequency. We show these normalized amplitudes for each experiment in Fig. 10a. As with Fig. 6 , the $1 / \omega$ line is plotted to show that APE follows the predicted scaling for the high-frequency range. This is in strong agreement with the amplitude characteristics obtained from the spectral analysis (Fig. 6). They differ only at the higher frequencies $(T<1 \mathrm{yr})$ in EKE because the gain calculated from the peaks in power spectral density is affected by the background slope of the power spectra. This background slope is due to natural variability, as demonstrated by the fact that it is also present in the steady wind experiment. We therefore consider the estimate of the forced response from the composite analysis to be the more robust one.

To characterize the phase of the response, the lag correlation of each ensemble member composite EKE and APE signal with the wind work is computed and averaged, giving the mean lag in each quantity for each experiment set. This lag (in days) for each experiment is then normalized by the corresponding forcing period to calculate the phase shift (in radians) in EKE and APE relative to the wind work (which is in phase with the wind stress by definition). The standard deviation in this lag correlation over all the ensemble members is used to represent the error in the lag calculation. The phase lag along with the error bars is shown in Fig. 10b.

In the limit of high forcing frequency, the error is much higher compared to the low-frequency cases. This is expected, since, as is evident from Fig. 9, the amplitude of oscillation is negligible for high forcing frequencies. As a result the lag correlation becomes statistically meaningless. While the mean phase lag in APE is zero at low frequency, it is out of phase by $\approx \pi / 2$ at the high-frequency limit. On the other hand, while EKE is shifted in phase by $\phi \approx \pi$ at the highfrequency limit, the phase difference approaches zero in the low-frequency range. Thus, the phase response characteristics of APE and EKE calculated from the numerical simulations are in accordance with the theoretical model.

The composites are also calculated for the barotropic and baroclinic parts of the zonal transport and are shown in Fig. 11. (The mean has been subtracted, since the mean barotropic and baroclinic transports differ greatly in magnitude.) The barotropic part does not exhibit 


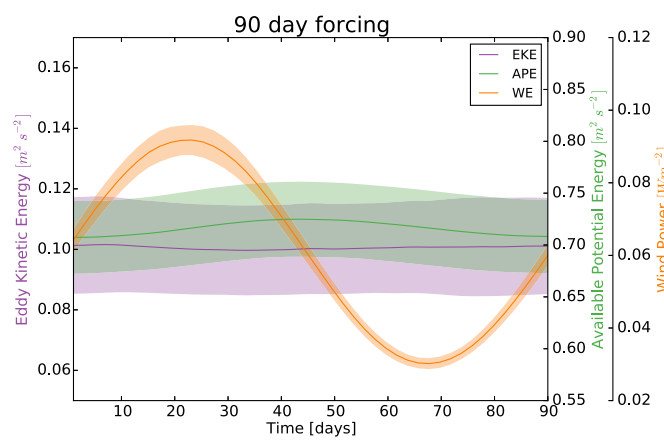

(a) $T=1 / 4$ year

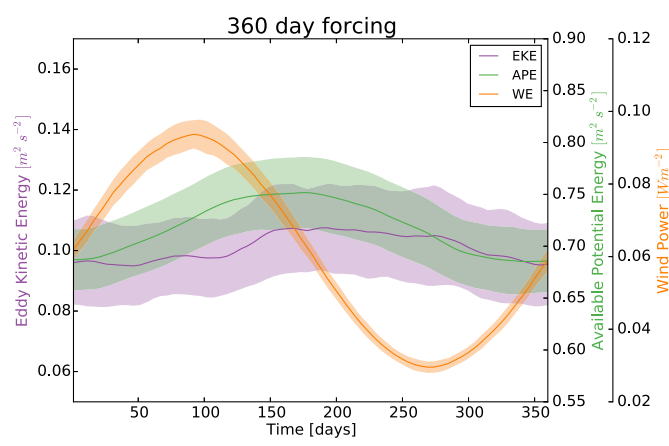

(c) $T=1$ year

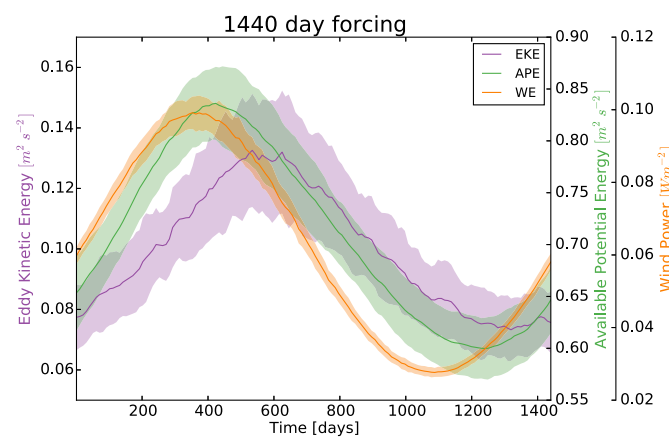

(e) $T=4$ years

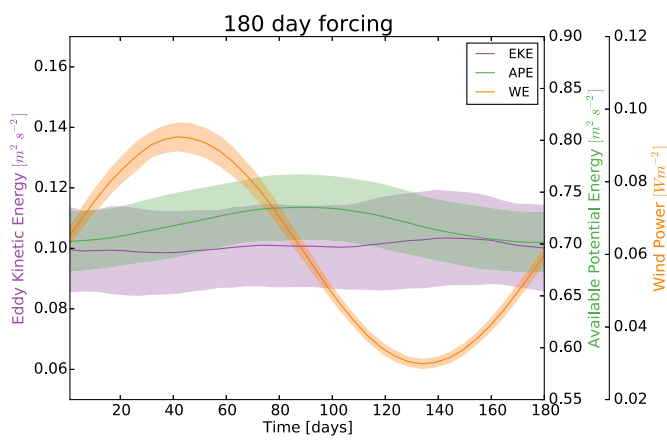

(b) $T=1 / 2$ year

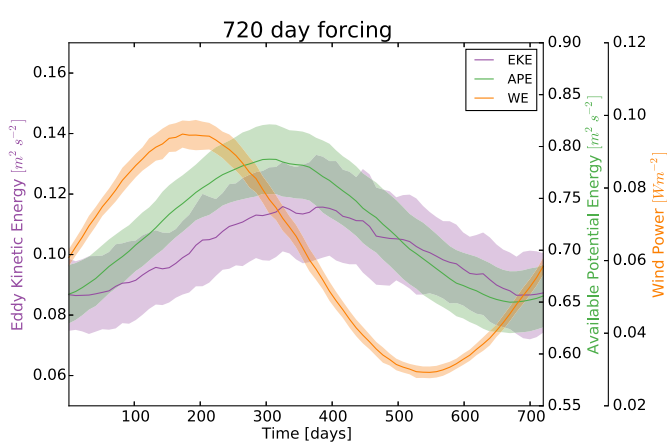

(d) $T=2$ years

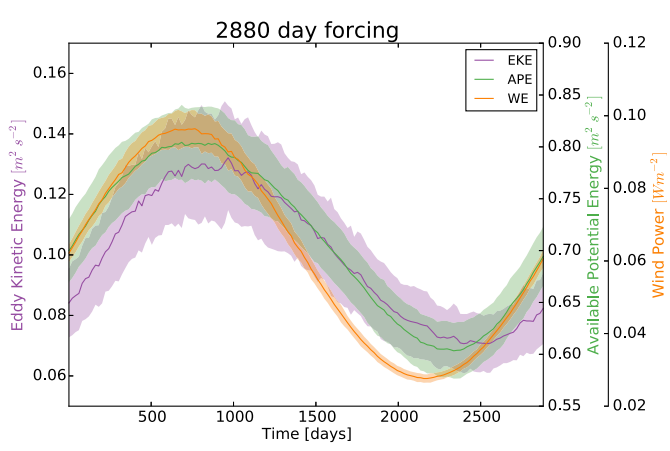

(f) $T=8$ years

FIG. 9. Composites of EKE, APE, and wind energy calculated over all ensemble members and all forcing cycles plotted as periodic signals with period corresponding to the external forcing frequency. Shading indicates the standard deviation. Note that for both APE and EKE, as the forcing frequency decreases, not only does the amplitude of oscillation increase but also approaches the wind work in phase.

significant periodicity, and its amplitude of oscillation remains fairly constant with forcing frequency. However, the baroclinic part oscillates more at lower forcing frequencies and also falls into phase with the wind work. A curious feature observed is that the envelope of variability in barotropic transport exhibits significant periodicity at lower frequencies.

In the next section, we revisit our simple analytical model and compare with our findings from the numerical simulations quantitatively.

\section{Comparing analytical model with numerical model}

In the analytical model derived in section 2 , the constants $k, c_{1}, c_{2}$, and $r$ can be evaluated from the steady-state balance. Examining (24) and (25), we identify four possible cases, depending on the choices for $\alpha$ and $\beta$ that we enumerate below.

\section{Case 1:}

For $\alpha=1 / 2$ and $\beta=3 / 2$ (weak eddy saturation and quadratic drag), 


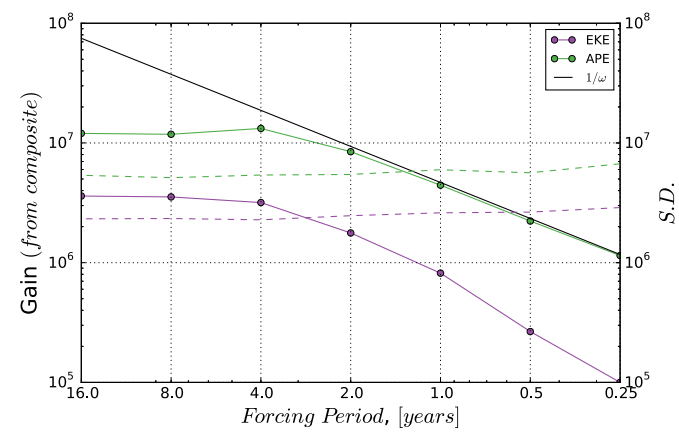

(a) Amplitude

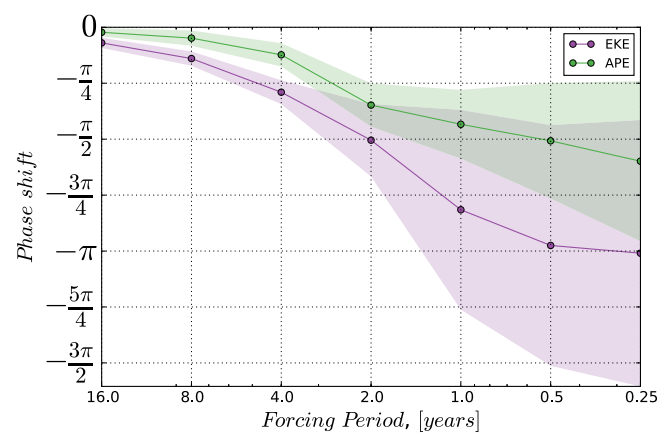

(b) Phase

FIG. 10. (a) The rms amplitude of oscillations of EKE and APE normalized by the rms amplitude of wind power plotted as a function of the forcing period shown alongside the standard deviation (noise floor) in each of these quantities (dashed lines) for the different ensemble experiments. (b) Phase shift of APE and EKE as a function of the forcing period with the standard deviation shown by shading.

$k=\frac{\bar{f}}{\bar{P} \overline{K^{1 / 2}}} r^{*}=\frac{k \bar{P}}{\bar{K}}, \quad c_{1}=k \bar{K}^{1 / 2}=\bar{f} / \bar{P}$,

$c_{2}=\frac{k \bar{P}}{2 \bar{K}^{1 / 2}}=\bar{f} / 2 \bar{K}, \quad$ and $\quad r=\frac{3 r^{*} \bar{K}^{1 / 2}}{2}=\frac{3 k \bar{P}}{2 \bar{K}^{1 / 2}}=3 \bar{f} / 2 \bar{K}$.

\section{Case 2:}

For $\alpha=1$ and $\beta=3 / 2$ (strong eddy saturation and quadratic drag),

$$
\begin{gathered}
k=\frac{\bar{f}}{\overline{P K}}, \quad r^{*}=\frac{k \bar{P}}{\bar{K}^{1 / 2}}, \quad c_{1}=k \bar{K}=\bar{f} / \bar{P}, \\
c_{2}=k \bar{P}=\bar{f} / \bar{K}, \quad \text { and } \quad r=\frac{3 r^{*} \bar{K}^{1 / 2}}{2}=\frac{3 k \bar{P}}{2}=3 \bar{f} / 2 \bar{K} .
\end{gathered}
$$

\section{Case 3:}

For $\alpha=1 / 2$ and $\beta=1$ (weak eddy saturation with linear drag),

$$
\begin{aligned}
& k=\frac{\bar{f}}{\bar{P} \overline{K^{1 / 2}}}, \quad r^{*}=\frac{k \bar{P}}{\bar{K}^{1 / 2}}, \quad c_{1}=k \bar{K}^{1 / 2}=\bar{f} / \bar{P}, \\
& c_{2}=\frac{k \bar{P}}{2 \bar{K}^{1 / 2}}=\bar{f} / 2 \bar{K}, \quad \text { and } \quad r=r^{*}=\frac{k \bar{P}}{\bar{K}^{1 / 2}}=\bar{f} / \bar{K} \text {. }
\end{aligned}
$$

\section{Case 4:}

For $\alpha=1$ and $\beta=1$ (strong eddy saturation with linear drag),

$$
\begin{aligned}
k & =\frac{\bar{f}}{\overline{P K}}, \quad r^{*}=k \bar{P}, \quad c_{1}=k \bar{K}=\bar{f} / \bar{P}, \\
c_{2} & =k \bar{P}=\bar{f} / \bar{K}, \quad \text { and } \quad r=r^{*}=k \bar{P}=\bar{f} / \bar{K} .
\end{aligned}
$$

Using the steady-state values of EKE, APE, and wind work calculated from the numerical experiments and using those values in (26) and (27), we can calculate the predicted gain and phase shift for APE and EKE. The constants $c_{1}, c_{2}$, and $r$ all have units of inverse time. For the range of forcing frequencies explored in our experiments, $c_{1}^{-1}=551 \pm 1$ days, while $c_{2}^{-1}=156 \pm 2$ days for $\alpha=1 / 2$ and $77 \pm 1$ days for $\alpha=1$. On the other hand, $r^{-1}=55 \pm 1$ days for quadratic drag and $79 \pm 1$ days for linear drag. These time scales provide a useful way of understanding the mechanisms governing the transient response.

As discussed in section 2, $c_{1}$ represents the linear baroclinic conversion (because of isopycnal slope changes), while $c_{2}$ represents the feedback of EKE on the conversion via its control over eddy mixing. Evidently the eddy feedback operates on a faster time scale than the simple slope change mechanism. This perhaps helps explain why models with constant Gent-McWilliams coefficients do not correctly reproduce the Southern Ocean response to changing winds (Gent 2016). This was demonstrated in Hofmann and Morales Maqueda (2011), wherein noneddy-resolving model simulations with constant and uniform GM eddy diffusivities were unable to appropriately represent eddy compensation. This result was further confirmed by analyzing a suite of ocean models using the CORE II forcing (Farneti et al. 2015), which showed that eddy compensation occurred only when the GM coefficient is variable. The fastest process, however, is still the frictional dissipation, which accounts for the smallamplitude response in EKE at high forcing frequencies; at a high forcing frequency, energy is removed by bottom friction before the eddy feedback can act.

Our numerical model implements a quadratic drag law, so, for the quantitative comparison, we only focus on cases 1 and 2 . We compare the results from the 


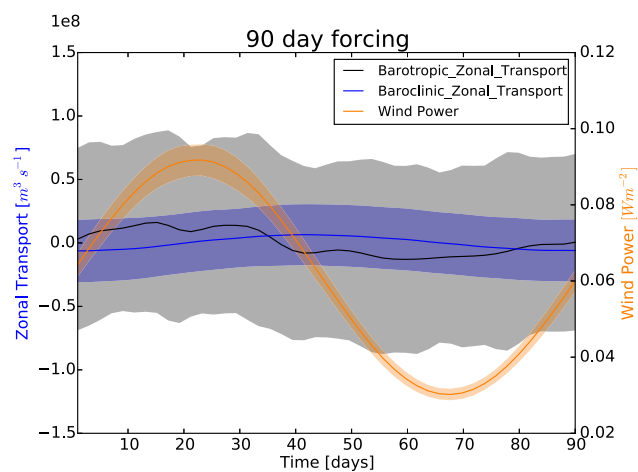

(a) $T=1 / 4$ year

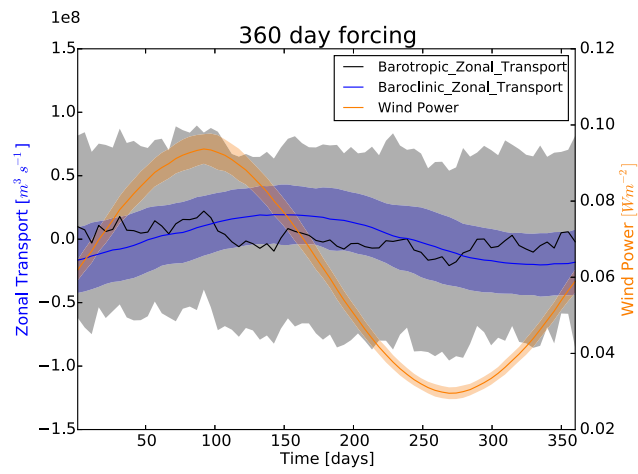

(c) $T=1$ year

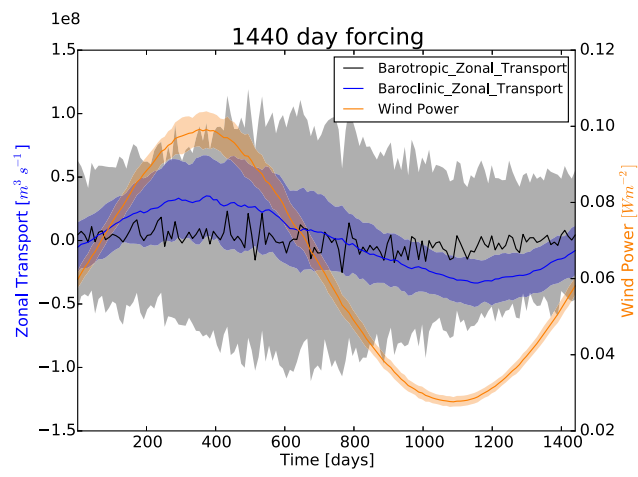

(e) $T=4$ years

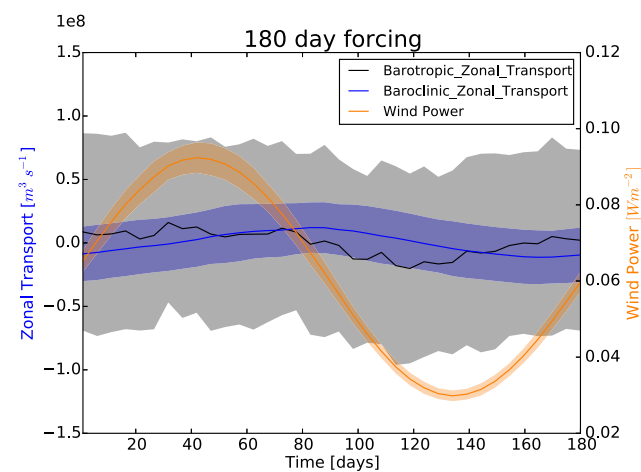

(b) $T=1 / 2$ year

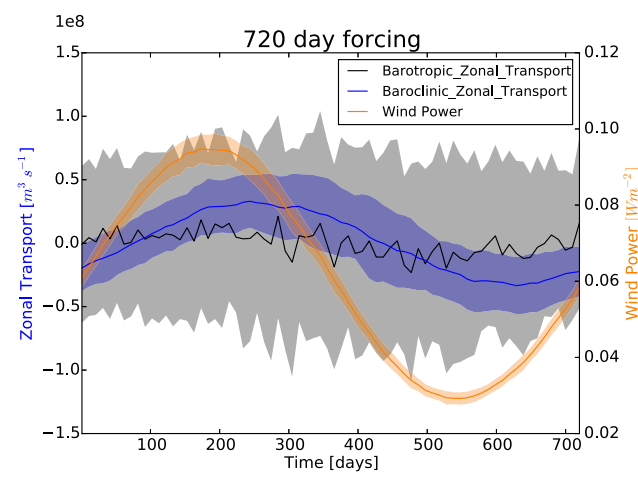

(d) $T=2$ years

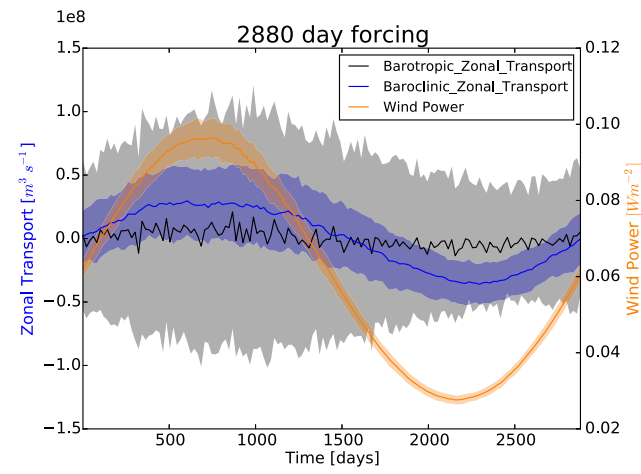

(f) $T=8$ years

FIG. 11. Composites of barotropic and baroclinic zonal transport calculated over all ensemble members and all forcing cycles plotted as periodic signals with period corresponding to the external forcing frequency. Both of these quantities are shown about a zero mean and where the shading represents the standard deviation. The wind power is also shown for easier phase comparison.

composite analysis with the theory using the parameter values derived as described above; the comparison is plotted in Fig. 12. The numerical simulation results agree quite well with the conceptual model in both gain and phase shift. The only place where we see some disagreement is at the high frequencies in EKE. However, it must be noted that, at high frequencies, the forced EKE oscillation amplitude is far below the noise floor of the internal variability. The fact that $\alpha=1$ gives the better agreement suggests the eddy feedback is even stronger than that predicted by the mixing length scaling $(\alpha=1 / 2)$ of (13). In the $\alpha=1$ solution, and in the numerical model results, APE gain displays weak maxima near the forcing period $T=4 \mathrm{yr}$. This agreement 

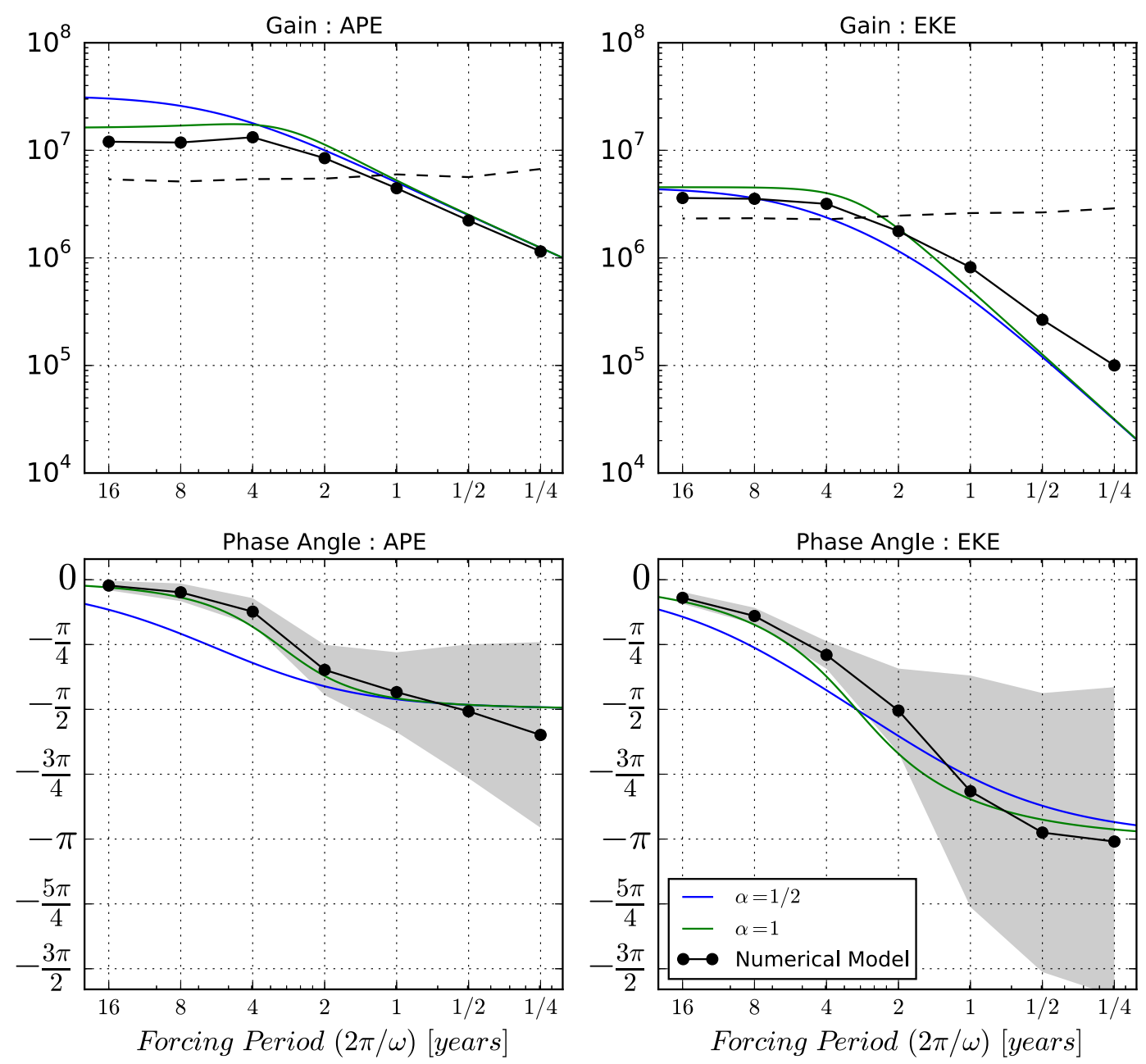

FIG. 12. Amplitude and phase of EKE and APE calculated for the two analytic cases: weak eddy feedback $(\alpha=$ $1 / 2$, blue) and strong eddy feedback ( $\alpha=1$, green) with quadratic drag. The values calculated from the numerical model (black) are shown alongside the ensemble standard deviation (black dashed lines for the gain, gray shading for phase angle). Note the excellent agreement between the analytical model and numerical simulations.

suggests that our analytical framework is capable of capturing rather subtle aspects of the periodically forced system.

\section{Discussion and conclusions}

We have explored the adiabatic Southern Ocean response to oscillating wind stress with the goal of understanding the time scales of mesoscale eddy equilibration. We have hypothesized that the transient ocean response lies between the two limits of Ekman response (when the time period of wind variability $T \leq 2 \mathrm{yr}$ ) and eddysaturated response (when $T \geq 8 \mathrm{yr}$ ), and this crossover time scale is effectively the time scale for eddy diffusive transport across the ACC $\left(\sim K_{\mathrm{GM}} / L_{y}^{2}\right)$. We developed a conceptual model of the energetic pathways between wind work, EKE, and APE and devised a transfer function characterizing the response of these variables to oscillatory winds as a function of forcing frequency. This hypothesis and conceptual energetic framework was tested using a suite of ensemble numerical simulations with an isopycnal model. We examined the power spectra of wind work, EKE, and APE and also used composite time series analysis to characterize the forced phase and amplitude response of these variables. We find that, in the high-frequency limit ( $T \leq$ $2 \mathrm{yr})$, the energy exchange is predominantly with APE, and the subsequent conversion to EKE is small. In this limit, mesoscale turbulence is too slow to efficiently remove energy input by the winds, which is stored as APE in the sloping isopycnals. At low frequencies $(T \geq 8 \mathrm{yr})$, the eddies are more efficient at 
extracting this energy. As a consequence, the ratio of the wind work converted to APE relative to EKE goes from 11 when $T=1 / 4 \mathrm{yr}$ to 3 when $T=16 \mathrm{yr}$. The phase characteristics indicate that, in the low-frequency limit, although the energy conversion to EKE is more efficient, the APE still leads EKE in phase. In the highfrequency limit, energy is transferred from wind work to APE with a phase lag of $\pi / 2$ by means of tilting isopycnals. At high frequencies, the EKE is predicted to be almost exactly out of phase with the wind stress, although the forced EKE response is too weak compared to the natural variability to reliably diagnose from the simulations.

The linearized framework for the energy pathway between wind work, APE, EKE, and dissipation reveals three constants, $c_{1}, c_{2}$, and $r$, which represent the characteristic inverse time scales of the three key processes involved. These three processes acting in conjunction determine the nature of the transient response; $c_{1}^{-1}$ is the linear baroclinic conversion time scale, $c_{2}^{-1}$ is the time scale of eddy feedback on the mixing, and $r^{-1}$ is the time scale of dissipation by frictional drag. Our numerical simulations are characterized by $c_{1}<c_{2}<r$.

The analytical model exhibits qualitatively different behavior for different combinations of $c_{1}, c_{2}$, and $r$. A special case occurs when $r=c_{2}$, in which the $K^{\prime}$ term cancels completely on the rhs of (25). In this unique case, EKE gain becomes independent of drag and is only governed by the conversion term $c_{1} P^{\prime}$, which does not depend on $\omega$. The APE response, on the other hand, has both $c_{1} P^{\prime}$ and $c_{2} K^{\prime}$ acting as sinks to remove energy. Since $c_{2} K^{\prime}$ remains strongly frequency dependent, the APE gain drops at low frequency, while EKE saturates (Fig. 3). This is one possible mechanism that can explain eddy saturation.

Of course, in a realistic context, it is unlikely that $r=$ $c_{2}$ exactly. However, similar behavior does occur when the two are close. Using the parameter values estimated from the numerical simulation assuming $\alpha=1$ (strong eddy feedback), we find $r=(53 \text { days })^{-1}$ and $c_{2}=$ $(78 \text { days })^{-1}$, which are relatively close compared to $c_{1}=$ (560 days $)^{-1}$. In the analytical solution for these parameters, we do observe a significant divergence between APE and EKE gain at low frequencies. This solution agrees with the numerical model response, which also shows reduced APE gain compared to EKE at low frequencies. We do not obtain such behavior from the analytical model when we assume $\alpha=1 / 2$ (weak eddy feedback). In this case, we estimate $r=$ $(53 \text { days })^{-1}$ and $c_{2}=(156 \text { days })^{-1}$, bringing the analytical solution closer to the model without eddy feedback at all (Fig. 2). Only when $c_{2}$ approaches $r$ do we see divergent behavior in APE and EKE gain. From this, we conclude that relatively strong eddy feedback is necessary to produce eddy saturation.

The zonal transport can be decomposed into a baroclinic part, directly related to APE via thermal wind, and a barotropic part. In the steady wind experiments, barotropic variability dominates the high frequencies, while the low-frequency variability is strongly baroclinic (Fig. 7). The forced response mirrors this division; lowfrequency forcing provokes a much stronger baroclinic response than barotropic response, while at high frequencies, the forced response is swamped by the internal barotropic variability. Like the APE, the baroclinic transport falls into phase with wind power at low frequencies. While the barotropic mode does not respond directly to low-frequency forcing, its variability (indicated by the error envelope in Fig. 11) does; this is likely associated with the periodic oscillations in EKE.

In this study, we have derived a transfer function that predicts the phase and amplitude response of EKE and APE to time-variable wind forcing. The constants $c_{1}, c_{2}$, and $r$ used to evaluate this transfer function were computed from the time-mean values of EKE, APE, and wind work diagnosed from an idealized numerical model. In future studies, we intend to extend this analysis to calculate the transfer function from realistic ocean models and observations of the Southern Ocean. In Fig. 1, we showed that the wind forcing signal over the Southern Ocean is composed of a broad spectrum of forcing frequencies of varying strength. The transfer function inferred from observations could be used to calculate the forced APE and EKE responses without the need to actually run an eddy-resolving model. This would represent a significant conceptual and computational advantage. More generally, the concept of transfer functions has wide applicability in climate studies (Hasselmann 1976; Laepple and Huybers 2014). Our approach could be applied to many different problems with time-variable forcing.

Our results also have implications for the parameterization of mesoscale eddies in coarse-resolution models. Out study, together with previous work with satellite altimetry and models (Meredith and Hogg 2006; Hogg et al. 2008; Meredith et al. 2012), shows that conversion from APE to EKE via baroclinic instability and dissipation of EKE by friction are separated in time. This separation strongly affects the transient response to changes in forcing. Between their generation and dissipation, finiteamplitude eddies can feed back on the conversion rate itself via their role in mixing. Most mesoscale parameterizations in use in global-scale ocean models, including those based on linear baroclinic growth rates (e.g., Visbeck et al. 1997; Danabasoglu and Marshall 2007), 
in contrast, will never capture this "memory effect" of mesoscale turbulence, which instead requires a timedependent reservoir of EKE. However, new parameterizations that prognostically model the subgrid EKE (e.g., Eden and Greatbatch 2008; Marshall and Adcroft 2010) would in theory fare much better in the scenario considered here. We therefore propose the problem of a channel flow forced by oscillating winds as an ideal test case for mesoscale parameterization.

A key ingredient in the Southern Ocean equilibration problem, which we have ignored in this study, is thermodynamics. The real ocean is, of course, not adiabatic. The Southern Ocean response to changing winds includes many thermodynamic factors such as the adjustment of surface turbulent and radiative heat fluxes and changes in sea ice cover (Ferreira et al. 2015). Furthermore, the thermodynamic forcing itself contains many different time scales, from the seasonal cycle in insolation to decadal-scale ozone depletion (Polvani and Smith 2013; Solomon et al. 2015). Combining all of these processes is only possible in a comprehensive global climate model. Our idealized modeling approach and conceptual model have shed light on one difficult aspect of the problem: the mesoscale eddy feedbacks on the isopycnal slope response to changes in winds. In the future, the transfer function approach could be used in conjunction with more comprehensive models to explore the role of thermodynamic processes associated with sea ice changes and ozone depletion, leading to a general and more complete theory for the variability of the Southern Ocean.

Acknowledgments. This work was supported by National Science Foundation Award OCE 1553593. A. S. received additional support from an NSF Frontiers in Earth System Dynamics (FESD) Grant OCE 1338814. The computations were carried out with highperformance computing support provided by NCAR's Computational and Information Systems Laboratory (CISL) and with Columbia University Information Technology (CUIT)'s Yeti High-Performance Computing (HPC) cluster service. Comments by Lorenzo Polvani and two anonymous reviewers greatly improved the manuscript.

\section{APPENDIX}

\section{Derivation of Analytical Formulation from Kinematics}

In section 2, we described a simple analytical model involving energetic pathways. Here, we derive the energy equations described by (1) and (2).
The inviscid, adiabatic, Boussinesq equations of motion are written as

$$
\begin{aligned}
\frac{D \mathbf{u}}{D t}+\mathbf{f} \times \mathbf{u} & =-\nabla \phi+\frac{1}{\rho_{0}} \nabla \tau, \\
\frac{D b}{D t} & =0, \\
\frac{\partial \phi}{\partial z} & =b, \quad \text { and } \\
\frac{\partial w}{\partial z} & =-\frac{\partial u}{\partial x}-\frac{\partial v}{\partial y} .
\end{aligned}
$$

We separate the flow into time and zonal mean $\overline{()}$ and a perturbation ()$^{\prime}$. Using this decomposition, the buoyancy equation for the background state may be written as

$$
\frac{\partial \bar{b}}{\partial t}=-\overline{\mathbf{u}} \cdot \nabla \bar{b}-\nabla \cdot \overline{\mathbf{u}^{\prime} b^{\prime}} .
$$

The perturbation buoyancy equation is written as

$\frac{\partial b^{\prime}}{\partial t}=-\overline{\mathbf{u}} \cdot \nabla b^{\prime}-\mathbf{u}^{\prime} \cdot \nabla \bar{b}-\nabla \cdot \mathbf{u}^{\prime} b^{\prime}+\nabla \cdot \overline{\mathbf{u}^{\prime} b^{\prime}}$.

Under quasigeostrophic scaling (Molemaker and McWilliams 2010), which is suitable for the conceptual model developed here, the APE density is given by $b^{2} / N^{2}$, where $\partial \bar{b} / \partial z=N^{2}$. This can be decomposed into a mean and a transient part as

$P=P_{m}+P_{t}=-\iiint_{d V}\left(\frac{\bar{b}^{2}}{2 N^{2}}\right)-\iiint_{d V}\left(\frac{b^{2}}{2 N^{2}}\right)$.

Note that $P_{m} \gg P_{t}$. Multiplying (A5) with $-\bar{b} / N^{2}$ and taking the global integral, we obtain the mean APE equation as

$$
\frac{d P_{m}}{d t}=\frac{d}{d t}\left(-\iiint_{d V} \frac{\bar{b}^{2}}{2 N^{2}}\right)=\iiint_{d V} \bar{w} \bar{b}-\iiint_{d V} \frac{\overline{\mathbf{u}^{\prime} b^{\prime}}}{\overline{N^{2}}} \cdot \nabla \bar{b}
$$

The first term is the conversion from background kinetic energy $\left(C_{K_{m} \rightarrow P_{m}}=\iiint_{d V} \bar{w} \bar{b}\right)$. The second term is the conversion to mean $\operatorname{APE}\left(C_{P_{m} \rightarrow P_{t}}=\iiint_{d V}\left[\overline{\mathbf{u}^{\prime} b^{\prime}} / N^{2}\right] \cdot \nabla \bar{b}\right)$. Using this, we rewrite (A8) as

$$
\frac{d P_{m}}{d t}=C_{K_{m} \rightarrow P_{m}}-C_{P_{m} \rightarrow P_{t}}
$$

Multiplying (A6) by $b^{\prime}$ and taking $\overline{()}$, we get the transient APE equation as 


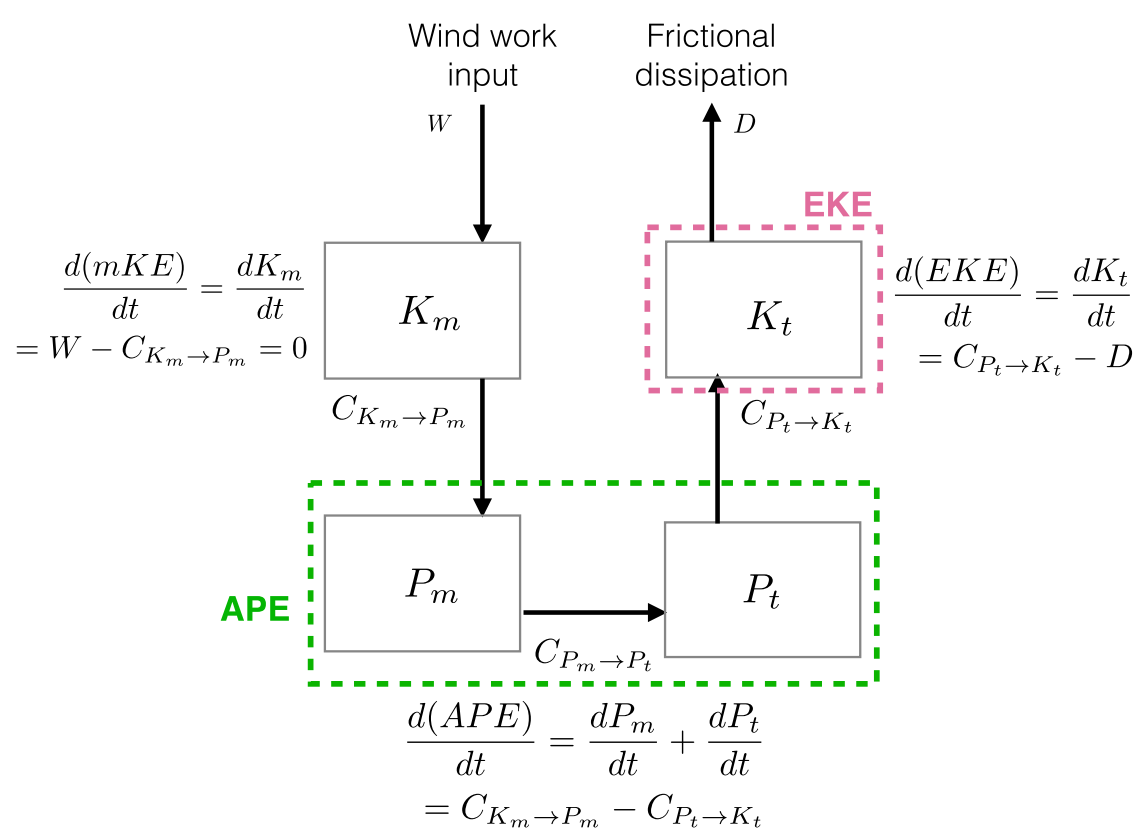

FIG. A1. Box model of the energy pathways involved in the simple analytical model derived in the appendix.

$$
\begin{aligned}
\frac{d P_{t}}{d t} & =\frac{d}{d t}\left(-\iiint_{d V} \overline{\frac{b^{\prime 2}}{2 N^{2}}}\right) \\
& =-\left(-\iiint_{d V} \overline{w^{\prime} b^{\prime}}\right)+\iiint_{d V} \frac{\overline{\mathbf{u}^{\prime} b^{\prime}}}{\overline{N^{2}}} \cdot \nabla \bar{b}
\end{aligned}
$$

The first term on the right is the conversion to transient (eddy) kinetic energy $\left(C_{P_{t} \rightarrow K_{t}}=-\iiint_{d V} \overline{w^{\prime} b^{\prime}}\right)$. The second term is the conversion from mean APE $\left(C_{P_{m} \rightarrow P_{t}}\right)$. Using this, we rewrite (A10):

$$
\frac{d P_{t}}{d t}=C_{P_{m} \rightarrow P_{t}}-C_{P_{t} \rightarrow K_{t}}
$$

Similarly the momentum equation for the background state is expressed as

$$
\frac{\partial \overline{\mathbf{u}}}{\partial t}+\overline{\mathbf{u}} \cdot \nabla \overline{\mathbf{u}}+\mathbf{f} \times \overline{\mathbf{u}}=-\nabla \bar{\phi}-\frac{1}{\rho_{0}} \frac{\partial \bar{\tau}}{\partial z}
$$

and the time varying part is

$$
\frac{\partial \mathbf{u}^{\prime}}{\partial t}+\left(\mathbf{u}^{\prime}+\overline{\mathbf{u}}\right) \cdot \nabla \mathbf{u}^{\prime}+\mathbf{u}^{\prime} \cdot \nabla \overline{\mathbf{u}}+\mathbf{f} \times \mathbf{u}^{\prime}=-\nabla \phi^{\prime}-\frac{1}{\rho_{0}} \frac{\partial \tau^{\prime}}{\partial z} .
$$

The equation for the evolution of mean kinetic energy, expressed as $K_{m}=(1 / 2) \iiint_{d V}(\overline{\mathbf{u}} \cdot \overline{\mathbf{u}})=(1 / 2) \iiint_{d V}\left(\bar{u}^{2}+\overline{\boldsymbol{v}}^{2}\right)$, is obtained by taking a dot product of (A8) with $\overline{\mathbf{u}}$. We drop the shear production by Reynolds stress, which is typically small in an oceanic context (Vallis 2006), and obtain

$$
\begin{aligned}
\frac{d K_{m}}{d t} & \simeq-\iiint_{d V} \overline{\mathbf{u}} \cdot \nabla \bar{\phi} \\
& =-\iiint_{d V} \bar{w} \bar{b}+\frac{1}{\rho_{0}} \iint_{d A}\left(\overline{\tau_{s}} \overline{u_{s}}-\overline{\tau_{b}} \overline{u_{b}}\right) .
\end{aligned}
$$

Here, the subscripts $s$ and $b$ denote surface and bottom, respectively. The second integral in (A14) is the "useful wind work" (Cessi et al. 2006; Abernathey et al. 2011). This is the amount of power available to drive baroclinic eddies. ${ }^{2}$

Continuing to neglect Reynolds stresses, the evolution equation for EKE, which is expressed as $K_{t}=$ $(1 / 2) \iiint_{d V}\left(\mathbf{u}^{\prime} \cdot \mathbf{u}^{\prime}\right)=(1 / 2) \iiint_{d V}\left(u^{\prime 2}+v^{\prime 2}\right)$, is obtained by taking a dot product of (A10) with $\mathbf{u}^{\prime}$ and taking $\overline{()}$ :

$$
\frac{d K_{t}}{d t} \simeq-\iiint_{d V} \overline{w^{\prime} b^{\prime}}-\frac{1}{\rho_{0}} \iint_{d A} \overline{\tau_{b}^{\prime} \cdot \mathbf{u}_{b}^{\prime}} .
$$

\footnotetext{
${ }^{2}$ From our numerical simulations, we actually computed the total wind work. A more accurate comparison would be obtained if we computed useful wind work only; unfortunately, the necessary variables were not saved for all the experiments. We did compute the useful wind work for some of the experiments, and for these experiments, $95 \%$ of the wind work was useful wind work. Also, given the very small amplitudes of oscillation in the barotropic transport (Fig. 11), and the already good agreement with the theory, we conclude that for the transient energy budget, the useful wind work is well approximated by the total wind work.
} 
The second term, which is the frictional drag $D$, can be written as $D \approx r_{b}\left(K_{t}\right)$ for the linear bottom drag. For quadratic frictional drag, this relation is replaced by $D \approx$ $C_{d}\left(K_{t}\right)^{3 / 2}$. Using these relations, the APE budget is written as

$$
\frac{d(\mathrm{APE})}{d t}=\frac{d\left(P_{m}\right)}{d t}+\frac{d\left(P_{t}\right)}{d t}=\text { wind work }-C_{P_{t} \rightarrow K_{t}},
$$

and the EKE equation is written as

$$
\frac{d(\mathrm{EKE})}{d t}=\frac{d\left(K_{t}\right)}{d t}=C_{P_{t} \rightarrow K_{t}}-D
$$

The mechanism of the energy transfer processes described above is illustrated in Fig. A1 with a box model.

In the context of our three-layer, reduced-gravity model, potential energy is evaluated as

$$
\mathrm{PE}=\frac{1}{2}\left\langle\left\langle\frac{\sum_{k=1}^{3} g_{k} \eta_{k}^{2}}{\sum_{k=1}^{3} h_{k}}\right\rangle\right\rangle .
$$

The eddy kinetic energy is defined as

$$
\mathrm{EKE}=\frac{1}{2}\left\langle\left\langle\frac{\sum_{k=1}^{3} h_{k}\left(u_{k}^{\prime 2}+v_{k}^{\prime 2}\right)}{\sum_{k=1}^{3} h_{k}}\right\rangle\right\rangle,
$$

where

$$
\langle\langle\cdot\rangle\rangle=\frac{1}{L_{x} L_{y}} \int_{0}^{L_{y}} d y \int_{0}^{L_{x}} d x(\cdot) .
$$

The subscripts denote the layers, $\eta$ is the interface height relative to mean sea level, $h$ is the layer thickness, and $g_{k}$ is the reduced gravity of layer $k$. All terms in the energy budget can be multiplied by $\rho_{0}$ to give units of watts per square meter.

\section{REFERENCES}

Abernathey, R., and P. Cessi, 2014: Topographic enhancement of eddy efficiency in baroclinic equilibration. J. Phys. Oceanogr., 44, 2107-2126, doi:10.1175/JPO-D-14-0014.1.

_ , and D. Ferreira, 2015: Southern Ocean isopycnal mixing and ventilation changes driven by winds. Geophys. Res. Lett., 42 , 10357-10365, doi:10.1002/2015GL066238.

_ J. Marshall, and D. Ferreira, 2011: The dependence of Southern Ocean meridional overturning on wind stress. J. Phys. Oceanogr., 41, 2261-2278, doi:10.1175/JPO-D-11-023.1.
—, D. Ferreira, and A. Klocker, 2013: Diagnostics of isopycnal mixing in a circumpolar channel. Ocean Modell., 72, 1-16, doi:10.1016/j.ocemod.2013.07.004.

Barnes, E. A., and L. Polvani, 2013: Response of the midlatitude jets, and of their variability, to increased greenhouse gases in the CMIP5 models. J. Climate, 26, 7117-7135, doi:10.1175/ JCLI-D-12-00536.1.

Boning, C. W., A. Dispert, M. Visbeck, S. R. Rintoul, and F. U. Schwarzkopf, 2008: The response of the Antarctic Circumpolar Current to recent climate change. Nat. Geosci., 1, 864869, doi:10.1038/ngeo362.

Cessi, P., W. R. Young, and J. A. Polton, 2006: Control of largescale heat transport by small-scale mixing. J. Phys. Oceanogr., 36, 1877-1894, doi:10.1175/JPO2947.1.

Chelton, D. B., R. A. deSzoeke, M. G. Schlax, K. El Naggar, and N. Siwertz, 1998: Geographical variability of the first baroclinic Rossby radius of deformation. J. Phys. Oceanogr., 28, 433-460, doi:10.1175/1520-0485(1998)028<0433:GVOTFB > 2.0.CO;2.

Chidichimo, M. P., K. A. Donohue, D. R. Watts, and K. L. Tracey, 2014: Baroclinic transport time series of the Antarctic Circumpolar Current measured in Drake Passage. J. Phys. Oceanogr., 44, 1829-1853, doi:10.1175/JPO-D-13-071.1.

Cunningham, S. A., S. G. Alderson, B. A. King, and M. A. Brandon, 2003: Transport and variability of the Antarctic Circumpolar Current in Drake Passage. J. Geophys. Res., 108, 8084, doi:10.1029/2001JC001147.

Danabasoglu, G., and J. Marshall, 2007: Effects of vertical variations of thickness diffusivity in an ocean general circulation model. Ocean Modell., 18, 122-141, doi:10.1016/j.ocemod.2007.03.006.

Dee, D., and Coauthors, 2011: The ERA-Interim reanalysis: Configuration and performance of the data assimilation system. Quart. J. Roy. Meteor. Soc., 137, 553-597, doi:10.1002/qj.828.

Eden, C., and R. J. Greatbatch, 2008: Towards a mesoscale eddy closure. Ocean Modell., 20, 223-239, doi:10.1016/j.ocemod.2007.09.002.

Farneti, R., and T. L. Delworth, 2010: The role of mesoscale eddies in the remote oceanic response to altered Southern Hemisphere winds. J. Phys. Oceanogr., 40, 2348-2354, doi:10.1175/ 2010JPO4480.1.

— polar Current and Southern Ocean meridional overturning circulation during 1958-2007 in a suite of interannual COREII simulations. Ocean Modell., 93, 84-120, doi:10.1016/ j.ocemod.2015.07.009.

Ferrari, R., and M. Nikurashin, 2010: Suppression of eddy diffusivity across jets in the Southern Ocean. J. Phys. Oceanogr., 40, 1501-1519, doi:10.1175/2010JPO4278.1.

Ferreira, D., J. Marshall, C. M. Bitz, S. Solomon, and A. Plumb, 2015: Antarctic Ocean and sea ice response to ozone depletion: A two-time-scale problem. J. Climate, 28, 1206-1226, doi:10.1175/JCLI-D-14-00313.1.

Gent, P. R., 2011: The Gent-McWilliams parameterization: 20/20 hindsight. Ocean Modell., 39, 2-9, doi:10.1016/ j.ocemod.2010.08.002.

_ 2016: Effects of Southern Hemisphere wind changes on the meridional overturning circulation in ocean models. Annu. Rev. Mar. Sci., 8, 79-94, doi:10.1146/annurev-marine-122414-033929.

_ , and J. C. McWilliams, 1990: Isopycnal mixing in ocean circulation models. J. Phys. Oceanogr., 20, 150-155, doi:10.1175/ 1520-0485(1990)020<0150:IMIOCM > 2.0.CO;2.

, J. Willebrand, T. J. McDougall, and J. C. McWilliams, 1995: Parameterizing eddy-induced tracer transports in ocean circulation models. J. Phys. Oceanogr., 25, 463-474, doi:10.1175/ 1520-0485(1995)025<0463:PEITTI >2.0.CO;2. 
Girod, B., R. Rabenstein, and A. Stenger, 2001: Signals and Systems. Wiley, $577 \mathrm{pp}$.

Grise, K. M., and L. M. Polvani, 2014: The response of midlatitude jets to increased $\mathrm{CO}_{2}$ : Distinguishing the roles of sea surface temperature and direct radiative forcing. Geophys. Res. Lett., 41, 6863-6871, doi:10.1002/2014GL061638.

Hallberg, R., 1997: Stable split time stepping schemes for largescale ocean modeling. J. Comput. Phys., 135, 54-65, doi:10.1006/ jcph.1997.5734.

_ , and A. Gnanadesikan, 2001: An exploration of the role of transient eddies in determining the transport of a zonally reentrant current. J. Phys. Oceanogr., 31, 3312-3330, doi:10.1175/ 1520-0485(2001)031<3312:AEOTRO > 2.0.CO;2.

— and _ 2006: The role of eddies in determining the structure and response of the wind-driven Southern Hemisphere overturning: Results from the Modeling Eddies in the Southern Ocean (MESO) project. J. Phys. Oceanogr., 36, 2232-2252, doi:10.1175/JPO2980.1.

Hasselmann, K., 1976: Stochastic climate models. Part I. Theory. Tellus, 28A, 473-485, doi:10.1111/j.2153-3490.1976.tb00696.x.

Henning, C. C., and G. K. Vallis, 2005: The effects of mesoscale eddies on the stratification and transport of an ocean with a circumpolar channel. J. Phys. Oceanogr., 35, 880-896, doi:10.1175/JPO2727.1.

Hofmann, M., and M. A. Morales Maqueda, 2011: The response of Southern Ocean eddies to increased midlatitude westerlies: A non-eddy resolving model study. Geophys. Res. Lett., 38, L03605, doi:10.1029/2010GL045972.

Hogg, A. M. C., W. K. Dewar, P. D. Killworth, and J. R. Blundell, 2003: A quasi-geostrophic coupled model (Q-GCM). Mon. Wea. Rev., 131, 2261-2278, doi:10.1175/1520-0493(2003)131<2261: AQCMQ $>2.0 . \mathrm{CO} ; 2$.

, M. P. Meredith, J. R. Blundell, and C. Wilson, 2008: Eddy heat flux in the Southern Ocean: Response to variable wind forcing. J. Climate, 21, 608-620, doi:10.1175/2007JCLI1925.1.

,-- , D. P. Chambers, E. P. Abrahamsen, C. W. Hughes, and A. K. Morrison, 2015: Recent trends in the Southern Ocean eddy field. J. Geophys. Res. Oceans, 120, 257-267, doi:10.1002/ 2014JC010470.

Holland, W. R., 1978: The role of mesoscale eddies in the general circulation of the ocean-Numerical experiments using a winddriven quasi-geostrophic model. J. Phys. Oceanogr., 8, 363-392, doi:10.1175/1520-0485(1978)008<0363:TROMEI $>2.0 . C O ; 2$.

Howard, E., A. M. Hogg, S. Waterman, and D. P. Marshall, 2015: The injection of zonal momentum by buoyancy forcing in a Southern Ocean model. J. Phys. Oceanogr., 45, 259-271, doi:10.1175/JPO-D-14-0098.1.

Johnson, G. C., and H. L. Bryden, 1989: On the size of the Antarctic Circumpolar Current. Deep-Sea Res., 36A, 39-53, doi:10.1016/ 0198-0149(89)90017-4.

Kalnay, E., and Coauthors, 1996: The NCEP/NCAR 40-Year Reanalysis Project. Bull. Amer. Meteor. Soc., 77, 437-471, doi:10.1175/1520-0477(1996)077<0437:TNYRP>2.0.CO;2.

Killworth, P. D., and M. M. Nanneh, 1994: Isopycnal momentum budget of the Antarctic Circumpolar Current in the Fine Resolution Antarctic Model. J. Phys. Oceanogr., 24, 1201-1223, doi:10.1175/1520-0485(1994)024<1201:IMBOTA > 2.0.CO;2.

Klocker, A., and R. Abernathey, 2014: Global patterns of mesoscale eddy properties and diffusivities. J. Phys. Oceanogr., 44, 1030-1046, doi:10.1175/JPO-D-13-0159.1.

, R. Ferrari, and J. H. LaCasce, 2012: Estimating suppression of eddy mixing by mean flows. J. Phys. Oceanogr., 42, 15661576, doi:10.1175/JPO-D-11-0205.1.
Laepple, T., and P. Huybers, 2014: Ocean surface temperature variability: Large model-data differences at decadal and longer periods. Proc. Natl. Acad. Sci. USA, 111, 16682-16687, doi:10.1073/pnas.1412077111.

Langlais, C. E., S. R. Rintoul, and J. D. Zika, 2015: Sensitivity of Antarctic Circumpolar Current transport and eddy activity to wind patterns in the Southern Ocean. J. Phys. Oceanogr., 45, 1051-1067, doi:10.1175/JPO-D-14-0053.1.

Lorenz, E. N., 1960: Energy and numerical weather prediction. Tellus, 12A, 364-373, doi:10.1111/j.2153-3490.1960.tb01323.x.

Marshall, D. P., and A. J. Adcroft, 2010: Parameterization of ocean eddies: Potential vorticity mixing, energetics and Arnold's first stability theorem. Ocean Modell., 32, 188-204, doi:10.1016/ j.ocemod.2010.02.001.

, D. R. Munday, L. C. Allison, R. J. Hay, and H. L. Johnson, 2016: Gills model of the Antarctic Circumpolar Current, revisited: The role of latitudinal variations in wind stress. Ocean Modell., 97, 37-51, doi:10.1016/j.ocemod.2015.11.010.

Marshall, G., 2003: Trends in the southern annular mode from observations and reanalyses. J. Climate, 16, 4134-4144, doi:10.1175/ 1520-0442(2003)016<4134:TITSAM $>2.0 . C O ; 2$.

Marshall, J., and T. Radko, 2003: Residual-mean solutions for the Antarctic Circumpolar Current and its associated overturning circulation. J. Phys. Oceanogr., 33, 2341-2354, doi:10.1175/ 1520-0485(2003)033<2341:RSFTAC $>2.0 . C O ; 2$.

Meredith, M. P., and A. M. Hogg, 2006: Circumpolar response of Southern Ocean eddy activity to a change in the southern annular mode. Geophys. Res. Lett., 33, L16608, doi:10.1029/ 2006 GL026499.

- , P. L. Woodworth, C. W. Hughes, and V. Stepanov, 2004: Changes in the ocean transport through Drake Passage during the 1980s and 1990s, forced by changes in the southern annular mode. Geophys. Res. Lett., 31, L21305, doi:10.1029/2004GL021169.

—, A. C. Naveira Garabato, A. M. Hogg, and R. Farneti, 2012: Sensitivity of the overturning circulation in the Southern Ocean to decadal changes in wind forcing. J. Climate, 25, 99 110, doi:10.1175/2011JCLI4204.1.

Millman, K. J., and M. Brett, 2007: Analysis of functional magnetic resonance imaging in python. Comput. Sci. Eng., 9, 52-55, doi:10.1109/MCSE.2007.46.

Molemaker, M. J., and J. C. McWilliams, 2010: Local balance and cross-scale flux of available potential energy. J. Fluid Mech., 645, 295-314, doi:10.1017/S0022112009992643.

Morrison, A. K., and A. M. Hogg, 2013: On the relationship between Southern Ocean overturning and ACC transport. J. Phys. Oceanogr., 43, 140-148, doi:10.1175/JPO-D-12-057.1.

Munday, D. R., and X. Zhai, 2015: Sensitivity of Southern Ocean circulation to wind stress changes: Role of relative wind stress. Ocean Modell., 95, 15-24, doi:10.1016/j.ocemod.2015.08.004.

- H. L. Johnson, and D. P. Marshall, 2013: Eddy saturation of equilibrated circumpolar currents. J. Phys. Oceanogr., 43, 507532, doi:10.1175/JPO-D-12-095.1.

Patara, L., C. W. Böning, and A. Biastoch, 2016: Variability and trends in Southern Ocean eddy activity in $1 / 12^{\circ}$ ocean model simulations. Geophys. Res. Lett., 43, 4517-4523, doi:10.1002/ 2016GL069026.

Pennel, R., and I. Kamenkovich, 2014: On the factors controlling the eddy-induced transport in the Antarctic Circumpolar Current. J. Phys. Oceanogr., 44, 2127-2138, doi:10.1175/ JPO-D-13-0256.1.

Polvani, L. M., and K. L. Smith, 2013: Can natural variability explain observed Antarctic sea ice trends? New modeling evidence from CMIP5. Geophys. Res. Lett., 40, 3195-3199, doi:10.1002/grl.50578. 
Qiu, B., S. Chen, P. Klein, H. Sasaki, and Y. Sasai, 2014: Seasonal mesoscale and submesoscale eddy variability along the North Pacific Subtropical Countercurrent. J. Phys. Oceanogr., 44, 3079-3098, doi:10.1175/JPO-D-14-0071.1.

Rintoul, S., and A. Naveira Garabato, 2013: Dynamics of the Southern Ocean circulation. Ocean Circulation and Climate: $A$ 21st Century Perspective, 2nd ed. G. Siedler et al., Eds., Academic Press, 471-492.

Smith, K. S., 2007: The geography of linear baroclinic instability in Earth's oceans. J. Mar. Res., 65, 655-683, doi:10.1357/ 002224007783649484

Solomon, A., L. M. Polvani, K. L. Smith, and R. P. Abernathey, 2015: The impact of ozone depleting substances on the circulation, temperature, and salinity of the Southern Ocean: An attribution study with CESM1(WACCM). Geophys. Res. Lett., 42, 5547-5555, doi:10.1002/2015GL064744.

Stammer, D., 1998: On eddy characteristics, eddy transports, and mean flow properties. J. Phys. Oceanogr., 28, 727-739, doi:10.1175/ 1520-0485(1998)028<0727:OECETA $>2.0 . C O ; 2$.

Swart, N. C., and J. C. Fyfe, 2012: Observed and simulated changes in the Southern Hemisphere surface westerly wind-stress. Geophys. Res. Lett., 39, L16711, doi:10.1029/2012GL052810.

Tandon, A., and C. Garrett, 1996: On a recent parameterization of mesoscale eddies. J. Phys. Oceanogr., 26, 406-411, doi:10.1175/ 1520-0485(1996)026<0406:OARPOM $>2.0 . \mathrm{CO} ; 2$.

Taschetto, A., I. Wainer, and M. Raphael, 2007: Interannual variability associated with semiannual oscillation in southern high latitudes. J. Geophys. Res., 112, D02106, doi:10.1029/2006JD007648.
Thomson, D., 1982: Spectrum estimation and harmonic analysis. Proc. IEEE, 70, 1055-1096, doi:10.1109/PROC.1982.12433. 2007: Jackknifing multitaper spectrum estimates. IEEE Signal Process. Mag., 24, 20-30, doi:10.1109/MSP.2007.4286561.

Treguier, A. M., J. Le Sommer, J. M. Molines, and B. de Cuevas, 2010: Response of the Southern Ocean to the southern annular mode: Interannual variability and multidecadal trend. J. Phys. Oceanogr., 40, 1659-1668, doi:10.1175/2010JPO4364.1.

Tulloch, R., J. Marshall, C. Hill, and K. S. Smith, 2011: Scales, growth rates, and spectral fluxes of baroclinic instability in the ocean. J. Phys. Oceanogr., 41, 1057-1076, doi:10.1175/ 2011JPO4404.1.

Vallis, G. K., 2006: Atmospheric and Oceanic Fluid Dynamics. Cambridge University Press, $745 \mathrm{pp}$

Viebahn, J., and C. Eden, 2010: Towards the impact of eddies on the response of the Southern Ocean to climate change. Ocean Modell., 34, 150-165, doi:10.1016/j.ocemod.2010.05.005.

Visbeck, M., J. Marshall, T. Haine, and M. Spall, 1997: Specification of eddy transfer coefficients in coarse-resolution ocean circulation models. J. Phys. Oceanogr., 27, 381-402, doi:10.1175/ 1520-0485(1997)027<0381:SOETCI $>2.0 . \mathrm{CO} ; 2$.

Ward, M. L., and A. M. Hogg, 2011: Establishment of momentum balance by form stress in a wind-driven channel. Ocean Modell., 40, 133-146, doi:10.1016/j.ocemod.2011.08.004.

Wilson, C., C. W. Hughes, and J. R. Blundell, 2015: Forced and intrinsic variability in the response to increased wind stress of an idealized Southern Ocean. J. Geophys. Res. Oceans, 120, 113-130, doi:10.1002/2014JC010315. 